TRANSACTIONS OF THE

AMERICAN MATHEMATICAL SOCIETY

Volume 364, Number 6, June 2012, Pages 2909-2932

S 0002-9947(2012)05426-2

Article electronically published on February 10, 2012

\title{
NUMBER OF CENTRAL CONFIGURATIONS AND SINGULAR SURFACES IN THE MASS SPACE IN THE COLLINEAR FOUR-BODY PROBLEM
}

\author{
TIANCHENG OUYANG AND ZHIFU XIE
}

\begin{abstract}
For a given $m=\left(m_{1}, m_{2}, \cdots, m_{n}\right) \in\left(\mathbf{R}^{+}\right)^{n}$, let $p$ and $q \in\left(\mathbf{R}^{d}\right)^{n}$ be two central configurations for $m$. Then we call $p$ and $q$ geometrically equivalent and write $p \sim q$ if they differ by a rotation followed by a scalar multiplication as well as by a permutation of bodies. Denote by $L(n, m)$ the set of geometric equivalence classes of $n$-body collinear central configurations for any given mass vector $m$. There are other different understandings of equivalence of central configurations in the collinear $n$-body problem. Under the usual definition of equivalence of central configurations in history, permutations of the bodies are not allowed, and we call them permutation equivalence. In this case Euler found three collinear central configurations and Moulton generalized to $n ! / 2$ central configurations for any given mass $m$ in the collinear $n$-body problem under permutation equivalence. In particular, the number of central configurations becomes from 12 under permutation equivalence to 1 under geometric equivalence for four equal masses in the collinear four-body problem. The main result in this paper is the discovery of the explicit parametric expressions of the union $\mathrm{H}_{4}$ of the singular surfaces in the mass space $m=\left(m_{1}, m_{2}, m_{3}, m_{4}\right) \in\left(\mathbf{R}^{+}\right)^{4}$, which decrease the number of collinear central configurations under geometric equivalence. We prove that the number of central configurations $\# L(4, m)=4 ! / 2-1=11$ if $m_{1}, m_{2}, m_{3}$ and $m_{4}$ are mutually distinct and $m \in H_{4}$.
\end{abstract}

\section{INTRODUCTION}

Some notation in this paper is similar to that in [15], 22] and [33]. For any $n \in \mathbf{N}$ (the set of integers), we denote by $P(n)$ the set of all permutations of $\{1,2, \cdots, n\}$. For any element $\tau \in P(n)$, we use $\tau=(\tau(1), \tau(2), \cdots, \tau(n))$ to denote the permutation $\tau$. We also denote a permutation of $m=\left(m_{1}, m_{2}, \cdots, m_{n}\right)$ by $m(\tau)=\left(m_{\tau(1)}, m_{\tau(2)}, \cdots, m_{\tau(n)}\right)$ for $\tau \in P(n)$. We define the converse permutation of $\tau$ by $\operatorname{con}(\tau)=(\tau(n), \cdots, \tau(1))$ and denote by ${ }^{\#} B$ the number of elements in a set $B$.

Received by the editors November 7, 2009 and, in revised form, January 13, 2010, April 4, 2010, and May 8, 2010.

2010 Mathematics Subject Classification. Primary 37N05, 70F10, 70F15, 37N30, 70H05, $70 \mathrm{~F} 17$.

Key words and phrases. Central configurations, super central configurations, $N$-body problem, geometric equivalence, permutation equivalence, mass equivalence, singular surfaces, relative equilibrium, Descartes' rule.

The second author was partially supported by RIG Grant (code 2137) from Virginia State University 2008-2009.

(C)2012 American Mathematical Society Reverts to public domain 28 years from publication 
Consider the Newtonian $n$-body problem:

$$
m_{k} \ddot{q}_{k}=\sum_{j=1, j \neq k}^{n} \frac{m_{k} m_{j}\left(q_{j}-q_{k}\right)}{\left|q_{j}-q_{k}\right|^{3}}=\frac{\partial U}{\partial q_{k}}, \quad 1 \leq k \leq n,
$$

where $U$ is the Newtonian potential function

$$
U=\sum_{1 \leq k<j \leq n} \frac{m_{k} m_{j}}{\left|q_{k}-q_{j}\right|} .
$$

Here $m_{k} \in \mathbf{R}^{+}$are the masses of the bodies and $q_{k} \in \mathbf{R}^{d}$ are their positions, respectively. Note that this potential function is positive, in contrast to the convention often used in introductory books. We will use $q \in\left(\mathbf{R}^{d}\right)^{n}$ and $m \in\left(\mathbf{R}^{+}\right)^{n}$ to denote the position and mass vectors $\left(q_{1}, \cdots, q_{n}\right)$ and $\left(m_{1}, \cdots, m_{n}\right)$, respectively. Let

$$
C=m_{1} q_{1}+\cdots+m_{n} q_{n}, \quad M=m_{1}+\cdots+m_{n}, \quad c=C / M
$$

be the first moment, total mass and center of mass of the bodies, respectively.

When we study homographic solutions of the $n$-body problem, the motion at any fixed time must satisfy the following nonlinear system of algebraic equations:

$$
\sum_{j=1, j \neq k}^{n} \frac{m_{j}\left(q_{j}-q_{k}\right)}{\left|q_{j}-q_{k}\right|^{3}}=-\lambda\left(q_{k}-c\right), \quad 1 \leq k \leq n,
$$

for a constant $\lambda$. By the homogeneity of $U(q)$ of degree -1 , we have $\lambda=U / 2 I>0$, where $I$ is the moment of inertial of the system, i.e. $I=\frac{1}{2} \sum_{i=1}^{n} m_{i}\left|q_{i}\right|^{2}$. The collision set is defined by

$$
\triangle=\bigcup\left\{q=\left(q_{1}, q_{2}, \cdots, q_{n}\right) \in\left(\mathbf{R}^{d}\right)^{n} \mid q_{i}=q_{j} \text { for some } i \neq j\right\} .
$$

To avoid singularities we will restrict $q$ to be in $V(n)$ :

$$
V(n)=\left\{q=\left(q_{1}, q_{2}, \cdots, q_{n}\right) \in\left(\mathbf{R}^{d}\right)^{n}\right\} \backslash \triangle .
$$

Definition 1.1 (Central configuration). A configuration $q \in V(n)$ is a central configuration (CC for short) for a given mass vector $m=\left(m_{1}, m_{2}, \cdots, m_{n}\right) \in$ $\left(\mathbf{R}^{+}\right)^{n}$ if $q$ is a solution of the system (2) for some constant $\lambda \in \mathbf{R}$.

Definition 1.2 (Geometric equivalence). Two configurations $q$ and $p \in V$ are $g e$ ometrically equivalent if they are similar modulo translations, dilations, rotations and permutations of the configuration. More precisely, $q$ and $p \in V$ are geometrically equivalent if there exist a vector $e \in \mathbf{R}^{d}$, a constant $\lambda \in \mathbf{R}$, a rotation $\mathcal{R} \in S O(d)$ and a permutation $\tau \in P(n)$ such that $q=(e, \lambda, \mathcal{R}, \tau) p$, i.e.,

$$
\left(q_{1}, q_{2}, \cdots, q_{n}\right)=\left(\lambda \mathcal{R}\left(p_{\tau(1)}-e\right), \lambda \mathcal{R}\left(p_{\tau(2)}-e\right), \cdots, \lambda \mathcal{R}\left(p_{\tau(n)}-e\right)\right) .
$$

In other words, the equivalent classes are the orbits of $\mathbf{R}^{d} \times \mathbf{R} \times S O(d) \times P(n)$ acting on $\left(\mathbf{R}^{d}\right)^{n}$.

It is easy to verify from the homogeneity and symmetry of (2) that any configuration which is equivalent to a central configuration is also a central configuration. One of the motivations for studying the geometric equivalence emanates from the existence of a special type of central configuration in [33, 34. There exists a configuration which is a central configuration for the order $\left(m_{1}, m_{2}, m_{3}, m_{4}\right)$ on a line and which is also a collinear central configuration for the permuted order $\left(m_{2}, m_{4}, m_{1}, m_{3}\right)$, where $m_{1}, m_{2}, m_{3}, m_{4}$ are distinct positive masses. These central configurations for the four distinct orders $\left(m_{1}, m_{2}, m_{3}, m_{4}\right),\left(m_{4}, m_{3}, m_{2}, m_{1}\right)$, 
$\left(m_{2}, m_{4}, m_{1}, m_{3}\right)$ and $\left(m_{3}, m_{1}, m_{4}, m_{2}\right)$ are equivalent under geometric equivalence. Therefore, the number of central configurations for such $m$ is less than or equal to $4 ! / 2-1=11$.

Sometimes it is more convenient and natural to speak of permutation equivalence classes, which are the same as above but without permutation. Under this usual definition of permutation equivalence of central configurations, collinear central configurations are one of a few families of central configurations with given positive masses which are sort of completely understood. For each way the particles can be ordered along a line, it is well known that there is a unique position that causes a central configuration. In this case, Euler discovered the collinear configurations for the three-body problem. Moulton 20] analyzed the general $n$-body case and proved that the number of central configurations in the collinear $n$-body problem is $n ! / 2$ for any $m \in \mathbf{R}^{+}$in 1910, and Smale [26] reconfirmed the result by a different variational approach in 1970. Historically, apart from the geometric equivalence and permutation equivalence, there is another way to define the equivalent classes in the collinear $n$-body problem, and it is called mass equivalence (see Definition 1.5). Because of these different understandings, the number of CCs were counted differently in different papers. A good review and discussion can be found in [15] and the reference therein. Long-Sun [15, 16] first addressed the problems, and they systematically studied collinear central configurations. They gave results on the enumerations of central configurations under each equivalence. Especially, in the sense of geometric equivalence, they found a singular algebraic hypersurface in the mass space which decreases the number of central configurations in the three-body problem. Xie 35 reinvestigated the collinear central configurations of the threebody problem, and he gave a direct parametric expression for the singular algebraic hypersurface in the mass space and a different proof of the main result of [15]. The number of central configurations of the collinear $n$-body problem in the sense of geometric equivalence for $n \geq 4$ is still unknown.

In this paper, our main goal is to study the collinear central configurations in the four-body problem in the sense of geometric equivalence. Without loss of generality, we suppose simply that the $n$ bodies are located on the $x$-axis. Then the set $V(n)$ of (44) can be modified to

$$
W(n)=\left\{q=\left(q_{1}, q_{2}, \cdots, q_{n}\right) \in \mathbf{R}^{n} \mid q_{1}<q_{2}<\cdots<q_{n}\right\} .
$$

Because in $W(n)$ we do not allow $q_{i}$ s to change their order, we now allow $m_{i}$ s to change their order. Note that when we say that $q=\left(q_{1}, \cdots, q_{n}\right) \in W(n)$ is a collinear CC for $m(\alpha) \equiv\left(m_{\alpha(1)}, \cdots, m_{\alpha(n)}\right)$ with some $\alpha \in P(n)$, we always mean that $m_{\alpha(i)}$ is put on $q_{i}$ for all $i=1, \cdots, n$. Then Definition 1.2 becomes Definition 1.3 .

Definition 1.3 (Geometric equivalence). Fix $m \in\left(\mathbf{R}^{+}\right)^{n}$ and let $q=\left(q_{1}, \cdots, q_{n}\right)$ and $p=\left(p_{1}, \cdots, p_{n}\right) \in W(n)$ be two collinear CCs for $m(\zeta)$ and $m(\eta)$ with $\zeta \in$ $P(n)$ and $\eta \in P(n)$. Then $(\zeta, q)$ and $(\eta, p)$ are geometrically equivalent, denoted by $q \sim p$, if either $q=a(p-b)$ or $q=a(\operatorname{con}(p)-b)$ for some $a \in \mathbf{R} \backslash\{0\}$ and $b=\left(b_{0}, b_{0}, \cdots, b_{0}\right) \in \mathbf{R}^{n}$. We denote by $L(n, m)$ the set of all geometric equivalence classes of $n$-body collinear central configurations for any given mass vector $m \in\left(\mathbf{R}^{+}\right)^{n}$.

Definition 1.4 (Permutation equivalence). Fix $m \in\left(\mathbf{R}^{+}\right)^{n}$ and let $q=\left(q_{1}, \cdots, q_{n}\right)$ and $p=\left(p_{1}, \cdots, p_{n}\right) \in W(n)$ be two collinear CCs for $m(\zeta)$ and $m(\eta)$ with $\zeta \in P(n)$ 
and $\eta \in P(n)$. Then $(\zeta, q)$ and $(\eta, p)$ are permutation equivalent, denoted by $q \sim_{P} p$, if $q \sim p$ and either $\zeta=\eta$ or $\zeta=c(\eta)$. We denote by $L_{P}(n, m)$ the set of all permutation equivalence classes of $n$-body collinear central configurations for any given mass vector $m \in\left(\mathbf{R}^{+}\right)^{n}$.

Definition 1.5 (Mass equivalence). Fix $m \in\left(\mathbf{R}^{+}\right)^{n}$ and let $q=\left(q_{1}, \cdots, q_{n}\right)$ and $p=\left(p_{1}, \cdots, p_{n}\right) \in W(n)$ be two collinear CCs for $m(\zeta)$ and $m(\eta)$ with $\zeta \in P(n)$ and $\eta \in P(n)$. Then $(\zeta, q)$ and $(\eta, p)$ are mass equivalent, denoted by $q \sim_{M} p$, if $q \sim p$ and either $m(\zeta)=m(\eta)$ or $m(\zeta)=m(c(\eta))$. We denote by $L_{M}(n, m)$ the set of all mass equivalence classes of $n$-body collinear central configurations for any given mass vector $m \in\left(\mathbf{R}^{+}\right)^{n}$.

Remark 1.6. (1) The geometric equivalence of two configurations in $V(n)$ or in $W(n)$ has nothing to do with central configurations or with masses. Generally speaking, geometric equivalence refers to the equivalence of geometric shapes. This is why it is called geometric equivalence.

(2) Definition 1.4 is the usual definition where the mass vector is attached to the position vector and the positions are not ordered on line. Most papers and books study the central configurations under permutation equivalence. Generally speaking, permutation of bodies makes a difference in permutation equivalence. This is why it is called permutation equivalence.

(3) As the results of [1, 2] for the four equal masses case, there are exactly 4 central configurations under geometric equivalence, i.e. the square, a special isosceles triangle with one body on its axis of the symmetry, an equilateral triangle with one body at its center, and a collinear central configuration. However, there are 50 central configurations for the four equal masses case under permutation equivalence. The unique collinear central configuration under geometric equivalence is counted $4 ! / 2=12$ central configurations under permutation equivalence because the permutation of the bodies on line makes a difference. This gives a good example of why we choose this terminology.

(4) Directly from the definitions, we can deduce that ${ }^{\#} L(n, m) \leq{ }^{\#} L_{M}(n, m) \leq \#$ $L_{P}(n, m)$. The fact that ${ }^{\#} L_{P}(n, m)=n ! / 2$ for any positive masses is proved by Moulton in 20] and by Smale in 27. ${ }^{\#} L_{M}(n, m)$ is already known in the papers 15, 16, 30, and ${ }^{\#} L(3, m)$ is studied by Long-Sun in [15. But ${ }^{\#} L(n, m)$ still remains open for $n \geq 4$.

We turn to study ${ }^{\#} L(4, m)$ for any given mass vector in the collinear four body problem. There exists a parametric surface $H_{4}$ (see Definition 2.3) in the mass space $\left(m_{1}, m_{2}, m_{3}, m_{4}\right) \in\left(\mathbf{R}^{+}\right)^{4}$ which decreases the number of collinear central configurations.

Theorem 1.7. For any mass vector $m=\left(m_{1}, m_{2}, m_{3}, m_{4}\right) \in\left(\mathbf{R}^{+}\right)^{4}$, one and only one of the following cases must apply:

(1) ${ }^{\#} L(4, m)={ }^{\#} L_{M}(4, m)=1$, if $m_{1}=m_{2}=m_{3}=m_{4}$;

(2) ${ }^{\#} L(4, m)={ }^{\#} L_{M}(4, m)=2$, if three of $m_{1}, m_{2}, m_{3}$, and $m_{4}$ are equal to each other but not the fourth;

(3) ${ }^{\#} L(4, m)={ }^{\#} L_{M}(4, m)=4$, if two pair of masses are equal but not all are equal;

(4) ${ }^{\#} L(4, m)={ }^{\#} L_{M}(4, m)=6$, if only one pair of masses is equal; 
(5) ${ }^{\#} L(4, m)={ }^{\#} L_{M}(4, m)-1=11$, if $m_{1}, m_{2}, m_{3}$ and $m_{4}$ are mutually distinct and $m \in H_{4}$;

(6) ${ }^{\#} L(4, m)={ }^{\#} L_{M}(4, m)=12$, if $m_{1}, m_{2}, m_{3}$ and $m_{4}$ are mutually distinct and $m \notin H_{4}$.

The question on the number of central configurations for a given mass vector $m=$ $\left(m_{1}, m_{2}, \cdots, m_{n}\right)$ is still a challenging problem for 21 st century mathematicians (see S. Smale [27]). The number of central configurations refers to the permutation equivalence classes in the references in this paragraph. Much progress has been made for four-body central configurations. The finiteness for the general four-body problem was settled by Hampton and Moeckel [ $[$. But an exact count is known only for the equal masses case [1, 2] where Albouy established a complete classification for the case of four equal masses by using Dziobek's coordinates and a symbolic computation program. Long and Sun [17] studied the convex central configurations with two pairs of equal masses on the opposite vertices, and they proved symmetry and uniqueness under some restrictions which were later removed by Perez-Chavela and Santoprete 23. Perez-Chavela and Santoprete proved that there is a unique convex noncollinear central configuration of the planar four-body problem when two equal masses are located at opposite vertices of a quadrilateral and, at most, only one of the remaining masses is larger than the equal masses. Leandro [13, 14] applied a combination of numerical and analytical methods to provide the solutions to the problem of central configuration for symmetrical classes or for one zero mass in the planar four-body problem. Based on numerical experiments, he used the method of rational parametrization and the method of resultants to give the exact numbers of central configurations for planar and spatial symmetrical classes. Bernat, Llibre and Pérez-Chavela 12 numerically studied the central configurations of the planar four-body problem with three equal masses. They observed that there is exactly one class of convex central configurations and there are one or two classes of concave central configurations. Shi and Xie 25] use analytic methods to show that besides the family of equilateral triangle configurations, there are exactly one family of concave and one family of convex central configurations of the planar four-body problem with three equal masses. Albouy, Fu and Sun 3] completely proved the uniqueness of planar four-body convex central configurations with only one pair of equal diagonal masses. They conjectured that, for any choice of four positive masses in the planar four-body problem, there exists a unique convex central configuration with given ordering of the particles. Also, in the same paper, they presented some conjectures for the spatial five-body central configurations with equal masses. Some partial results of central configurations are given in [12, 17, 28, 25, 32, for the four-body problem with some equal masses, in [7, 8, 9, 10, 29, 36, for the five or more body problem, and in [5, 31] for general homogenous or quasi-homogeneous potentials. Recently, the central configurations in the collinear $n$-body problem were reinvestigated in [4, 22, 31, 33. and some new phenomenons were observed. For the importance and more properties of central configurations and related topics, we refer to the works of R. Moeckel [21, D. Saari [24, and the books [18, 19]. There are more papers which studied central configurations of the four-body problem, and you can find them from the references therein.

Our paper is organized as follows. In section 2, we describe the total singular surface $H_{4}$ and its properties. The proof for the main theorem, Theorem 1.7 
is conducted in section 3 . Section 4 carries out the proof for some lemmas and propositions which are used in the proof of the main theorem.

\section{The total Singular surface $H_{4}$}

In this section, we describe the total singular surface $H_{4}$ and some propositions which are to be used in the proof of the main theorem. We first denote the polynomials $f_{i}(s, t), i=1,2, \cdots, 5$ by

$$
\begin{gathered}
f_{1}(s, t)=(s+t+2)^{2}(s+2)^{2} s^{2}\left(t^{5}+5 t^{4}+8 t^{3}-4 t^{2}-16 t-16\right), \\
f_{2}(s, t)=(s+t+2)^{2}(s+2)^{2} s^{2}\left(t^{4}+4 t^{3}+4 t^{2}+16 t+16\right), \\
f_{3}(s, t)=4(t+2)^{2} s^{2}\left(s^{5}+9 s^{4}+32 s^{3}+56 s^{2}+48 s+16\right. \\
+\left(2 s^{4}+14 s^{3}+36 s^{2}+40 s+16\right) t \\
\left.+\left(s^{3}+5 s^{2}+8 s+4\right) t^{2}+\left(-s^{2}-8-6 s\right) t^{3}+(-2 s-5) t^{4}-t^{5}\right), \\
f_{4}(s, t)=4(t+2)^{2} s^{2}\left(s^{4}+8 s^{3}+24 s^{2}+32 s+16+\left(2 s^{3}+12 s^{2}+24 s+16\right) t\right. \\
\left.+\left(s^{2}+4 s+4\right) t^{2}+(2 s+4) t^{3}+t^{4}\right), \\
f_{5}(s, t)=16(s+2)^{4}+16(s+4)(s+2)^{3} t+4\left(s^{2}+12 s+24\right)(s+2)^{2} t^{2} \\
+4(s+2)\left(s^{3}+2 s^{2}+12 s+16\right) t^{3}+\left(s^{4}+4 s^{3}+4 s^{2}+16 s+16\right) t^{4} .
\end{gathered}
$$

Note that $f_{5}(s, t)=f_{5}(t, s)$. For the sake of convenience, we let

$$
f_{i i}(s, t)=f_{i}(t, s), \quad i=1,2,3,4 .
$$

$f_{i}$ and $f_{i i}$ always mean $f_{i}(s, t)$ and $f_{i i}(s, t)$. They have the following properties.

Proposition 2.1 (Lemma 3.1, Z. Xie, [33). For any $s>0, t>0$,

$$
\begin{gathered}
f_{2}>f_{4}>0, f_{22}>f_{44}>0, \\
f_{2}-f_{4}+f_{44}-f_{22}=0, \\
f_{1}+f_{11}+f_{3}+f_{33}>0, \\
f_{2}=f_{22}, f_{4}=f_{44} \text { if and only if } s=t .
\end{gathered}
$$

To define $H_{4}$, we need to introduce an algebraic curve $\bar{\Gamma}_{31}$ in the first quadrant of the st-plane.

Definition 2.2. Let

$$
\begin{aligned}
& g_{31}(s, t)=-f_{33} f_{2}{ }^{2}-f_{33} f_{44} f_{4}+f_{44} f_{2} f_{1}+f_{44}^{2} f_{1}-f_{44} f_{2} f_{33}-f_{44}^{2} f_{3} \\
& +f_{11}{f_{2}}^{2}+f_{11} f_{44} f_{4}-f_{22} f_{3} f_{2}-f_{22} f_{4} f_{1}+f_{22} f_{4} f_{33}+f_{22} f_{2} f_{1}
\end{aligned}
$$

and

$$
\begin{gathered}
\Gamma_{31}=\left\{(s, t) \mid g_{31}(s, t)=0, s>0, t>0\right\}, \\
\bar{\Gamma}_{31}=\left\{(s, t) \in \Gamma_{31} \mid s_{0}<s, t<s_{1}\right\},
\end{gathered}
$$

where $s_{0}, s_{1}$ are constant and are determined later in Proposition 2.5. Numerically $s_{0}=1.297093169, s_{1}=3.086044724$ (see Figure 1). Let $\bar{s}$ be the unique positive root of the polynomial,

$$
s^{7}+7 s^{6}+19 s^{5}-84 s^{3}-152 s^{2}-112 s-32=0,
$$




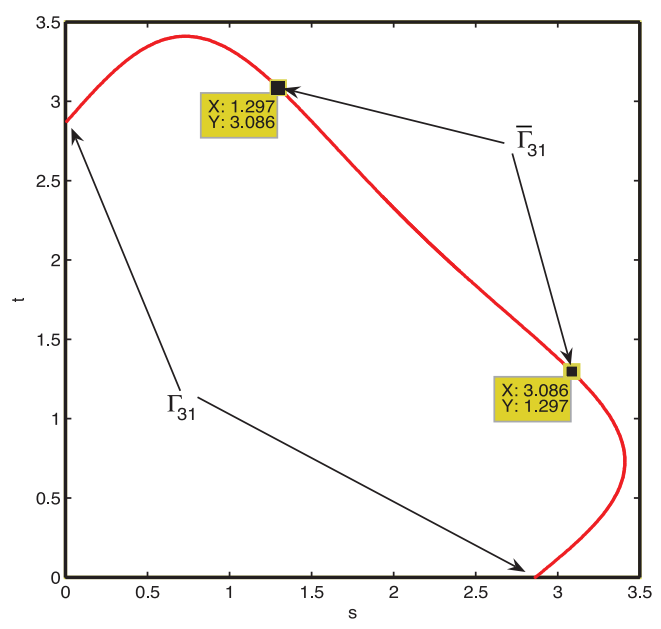

Figure 1. Singular curve $\Gamma_{31}$ and $\bar{\Gamma}_{31}$ in the st-plane.

and numerically $\bar{s}=2.162120398$. We call $g_{31}$ and $\bar{\Gamma}_{31}$ the singular polynomial and singular curve.

To understand the singular curve $\bar{\Gamma}_{31}$, note that by Descartes' rule of signs (cf. p. 300 of [11), the equation $g_{31}(s, t)=0$ defines an implicit function of $s$ for $0<s<\bar{s}$ and an implicit function of $t$ for $0<t<\bar{s}$. Further details on properties of the singular curve $\bar{\Gamma}_{31}$ are given in Proposition 2.5 below.

$P(4)$ has 24 permutations. They are defined by

$$
\begin{array}{lcrl}
\tau_{1}=(1,2,3,4), & \tau_{2}=(1,2,4,3), & \tau_{3}=(1,3,2,4), \\
\tau_{4}=(1,3,4,2), & \tau_{5}=(1,4,2,3), & \tau_{6}=(1,4,3,2), \\
\tau_{7}=(2,1,3,4), & \tau_{8}=(2,1,4,3), & \tau_{9}=(2,3,1,4), \\
\tau_{10}=(2,4,1,3), & \tau_{11}=(3,1,2,4), & \tau_{12}=(3,2,1,4)
\end{array}
$$

and their converse permutations $\operatorname{con}\left(\tau_{i}\right), i=1, \cdots, 12$.

Definition 2.3 (Singular surfaces in mass space). Let $t=t(s)$ be the function implicitly defined by $g_{31}(s, t)=0$ for $s_{0}<s<s_{1}$. For $i=1,2,3,4$, let $\varphi_{i}(\lambda, s)$ be a function with two variables $(\lambda, s)$ given by

$$
\begin{aligned}
& \varphi_{1}(\lambda, s)=\frac{\lambda\left(f_{1} f_{44} f_{4}-f_{2} f_{44} f_{1}+f_{2}{ }^{2} f_{33}+f_{2} f_{44} f_{3}\right)}{\left(f_{2}{ }^{2}+f_{44} f_{4}\right) f_{5}}, \\
& \varphi_{2}(\lambda, s)=\frac{\lambda\left(f_{3} f_{2}{ }^{2}+f_{4} f_{2} f_{1}+f_{1} f_{44} f_{4}-f_{4} f_{2} f_{33}\right)}{\left(f_{2}{ }^{2}+f_{44} f_{4}\right) f_{5}}, \\
& \varphi_{3}(\lambda, s)=\frac{\lambda\left(f_{2}{ }^{2} f_{33}+f_{33} f_{44} f_{4}-f_{2} f_{44} f_{1}-f_{44}{ }^{2} f_{1}+f_{44} f_{2} f_{33}+f_{44}{ }^{2} f_{3}\right)}{\left(f_{2}{ }^{2}+f_{44} f_{4}\right) f_{5}}, \\
& \varphi_{4}(\lambda, s)=-\frac{\lambda\left(-f_{11} f_{2}{ }^{2}-f_{11} f_{44} f_{4}-f_{22} f_{2} f_{1}-f_{22} f_{44} f_{1}+f_{22} f_{2} f_{33}+f_{22} f_{44} f_{3}\right)}{\left(f_{2}{ }^{2}+f_{44} f_{4}\right) f_{5}},
\end{aligned}
$$


where $t$ is given by the function $t=t(s), 0<\lambda<\infty, s_{0}<s<s_{1}$, and $s \neq \bar{s}$. We define the following parametric surfaces in positive quadrant $m=\left(m_{1}, m_{2}, m_{3}, m_{4}\right) \in$ $\left(\mathbf{R}^{+}\right)^{4}$ by

(i) $\psi_{11}=\left\{\left(m_{1}, m_{2}, m_{3}, m_{4}\right) \mid m_{i}(\lambda, s)=\varphi_{\tau_{1}(i)}(\lambda, s), i=1,2,3,4\right\}$;

$\psi_{12}=\left\{\left(m_{1}, m_{2}, m_{3}, m_{4}\right) \mid m_{i}(\lambda, s)=\varphi_{\tau_{10}(i)}(\lambda, s), i=1,2,3,4\right\} ;$

$\Psi_{1}=\psi_{11} \bigcup \psi_{12}$.

(ii) $\psi_{21}=\left\{\left(m_{1}, m_{2}, m_{3}, m_{4}\right) \mid m_{i}(\lambda, s)=\varphi_{\tau_{2}(i)}(\lambda, s), i=1,2,3,4\right\}$;

$\psi_{22}=\left\{\left(m_{1}, m_{2}, m_{3}, m_{4}\right) \mid m_{i}(\lambda, s)=\varphi_{\tau_{9}(i)}(\lambda, s), i=1,2,3,4\right\} ;$

$\Psi_{2}=\psi_{21} \bigcup \psi_{22}$.

(iii) $\psi_{31}=\left\{\left(m_{1}, m_{2}, m_{3}, m_{4}\right) \mid m_{i}(\lambda, s)=\varphi_{\tau_{3}(i)}(\lambda, s), i=1,2,3,4\right\}$;

$\psi_{32}=\left\{\left(m_{1}, m_{2}, m_{3}, m_{4}\right) \mid m_{i}(\lambda, s)=\varphi_{\tau_{8}(i)}(\lambda, s), i=1,2,3,4\right\} ;$

$\Psi_{3}=\psi_{31} \bigcup \psi_{32}$.

(iv) $\psi_{41}=\left\{\left(m_{1}, m_{2}, m_{3}, m_{4}\right) \mid m_{i}(\lambda, s)=\varphi_{\tau_{4}(i)}(\lambda, s), i=1,2,3,4\right\}$;

$\psi_{42}=\left\{\left(m_{1}, m_{2}, m_{3}, m_{4}\right) \mid m_{i}(\lambda, s)=\varphi_{\tau_{12}(i)}(\lambda, s), i=1,2,3,4\right\} ;$

$\Psi_{4}=\psi_{41} \bigcup \psi_{42}$.

(v) $\psi_{51}=\left\{\left(m_{1}, m_{2}, m_{3}, m_{4}\right) \mid m_{i}(\lambda, s)=\varphi_{\tau_{5}(i)}(\lambda, s), i=1,2,3,4\right\}$;

$\psi_{52}=\left\{\left(m_{1}, m_{2}, m_{3}, m_{4}\right) \mid m_{i}(\lambda, s)=\varphi_{\tau_{7}(i)}(\lambda, s), i=1,2,3,4\right\} ;$

$\Psi_{5}=\psi_{51} \bigcup \psi_{52}$.

(vi) $\psi_{61}=\left\{\left(m_{1}, m_{2}, m_{3}, m_{4}\right) \mid m_{i}(\lambda, s)=\varphi_{\tau_{6}(i)}(\lambda, s), i=1,2,3,4\right\}$;

$\psi_{62}=\left\{\left(m_{1}, m_{2}, m_{3}, m_{4}\right) \mid m_{i}(\lambda, s)=\varphi_{\tau_{11}(i)}(\lambda, s), i=1,2,3,4\right\} ;$

$\Psi_{6}=\psi_{61} \bigcup \psi_{62}$.

$$
H_{4}=\bigcup_{i=1}^{6} \Psi_{i} .
$$

We call $\Psi_{i}$ for $1 \leq i \leq 6$ and $H_{4}$ the singular surface and the total singular surface, respectively.

Remark 2.4 (Remarks on the functions $\varphi_{i}$ and $\Psi_{i}$ ). First note that $\varphi_{i}(\lambda, s)$ is a linear function on $\lambda$. The geometric equivalence is created only by positions instead of the parameter $\lambda$. The linearity of $\varphi_{i}$ on $\lambda$ reflects the fact that the number of central configurations for proportional two sets of masses are the same. $\varphi_{i}$ comes from the proof of Lemma 3.6. The graph of $\varphi_{i}$ for $i=1,2,3,4$ is shown in Figure2. We prove that $\varphi_{3}>\varphi_{1}>\varphi_{4}>\varphi_{2}>0$ for $s_{0}<s<\bar{s}, \varphi_{2}>\varphi_{4}>\varphi_{1}>\varphi_{3}>0$ for $\bar{s}<s<s_{1}$, and $\varphi_{1}=\varphi_{2}=\varphi_{3}=\varphi_{4}$ at $s=\bar{s}$ in Proposition 2.5. So the intersection of $\Psi_{i}$ and $\Psi_{j}$ is empty for any $1 \leq i \neq j \leq 6$. The proof of Proposition 2.5 can easily be adapted from the proof of Proposition 2.5 in [33].

Proposition 2.5. Properties of $\Gamma_{31}$ and $\bar{\Gamma}_{31}$ :

(i) $g_{31}(s, t)=g_{31}(t, s)$ holds for all $(s, t) \in\left(\mathbf{R}^{+}\right)^{2}$. Then the points on $g_{31}(s, t)$ $=0$ are symmetric about $s=t$.

(ii) The unique intersection point of $g_{31}(s, t)=0$ and $s=t$ is $(\bar{s}, \bar{s})$, given by the unique positive root of the polynomial:

$$
s^{7}+7 s^{6}+19 s^{5}-84 s^{3}-152 s^{2}-112 s-32=0,
$$

and numerically $\bar{s}=2.162120398$.

(iii) For $0<s<\bar{s}, t$ is a continouous function of $s$ implicitly defined by $g_{31}(s, t)=0$. For $0<t<\bar{s}$, $s$ is a continouous function of $t$ implicitly defined by $g_{31}(s, t)=0$. Furthermore the curve $\Gamma_{31}=\left\{(s, t) \mid g_{31}(s, t)=0\right\}$ is in the region $\{(s, t) \mid 0<s<4,0<t<4\}$ (see Figure 1). 


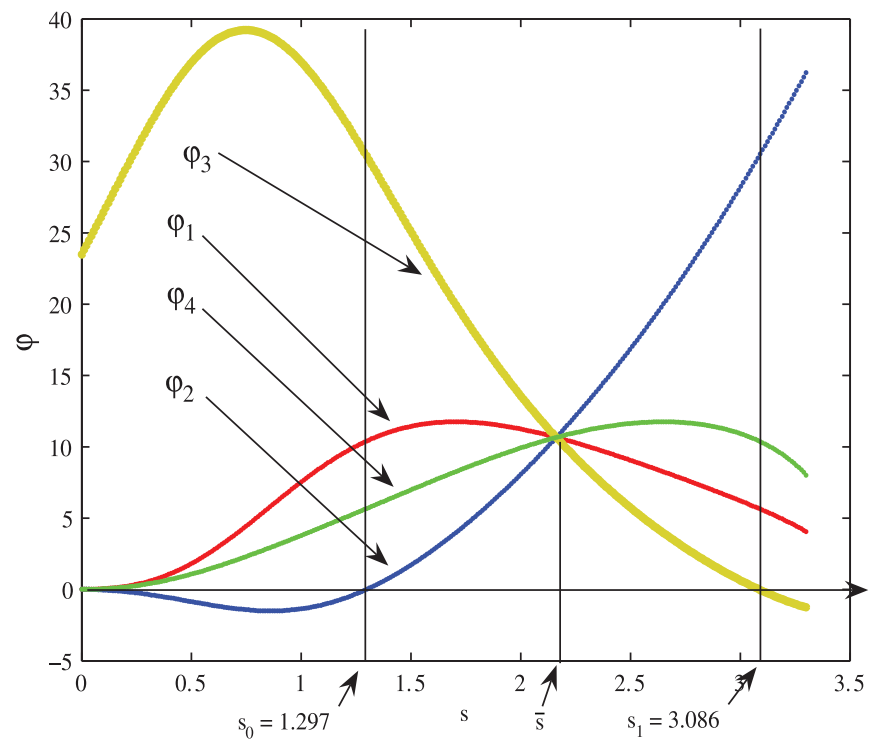

Figure 2. Let $\lambda=1$. The graph of $\varphi_{i}(\lambda, s)$ given by (16) along $\bar{\Gamma}_{31} \cdot \varphi_{3}>\varphi_{1}>\varphi_{4}>\varphi_{2}>0$ for $s_{0}<s<\bar{s}, \varphi_{2}>\varphi_{4}>\varphi_{1}>\varphi_{3}>$ 0 for $\bar{s}<s<s_{1}$, and $\varphi_{1}=\varphi_{2}=\varphi_{3}=\varphi_{4}$ at $s=\bar{s}$.

(iv) If $\varphi=\left(\varphi_{1}, \varphi_{2}, \varphi_{3}, \varphi_{4}\right)$ is given by (16), then $\varphi_{i}>0(i=1,2,3,4)$ along the curve

$$
\bar{\Gamma}_{31}=\left\{(s, t) \in \Gamma_{31} \mid s_{0}<s, t<s_{1}\right\},
$$

where $\left(s_{0}, t_{0}\right)$ is the intersection point of $\varphi_{2}=0$ and $g_{31}=0$ and where $\left(s_{1}, t_{1}\right)$ is the intersection point of $\varphi_{3}=0$ and $g_{31}=0$. Numerically $s_{0}$ $=1.297093169, s_{1}=3.086044724, t_{0}=s_{1}$, and $t_{1}=s_{0}$. Furthermore, for $s_{0}<s<\bar{s}, \varphi_{3}>\varphi_{1}>\varphi_{4}>\varphi_{2}$, and for $\bar{s}<s<s_{1}, \varphi_{2}>\varphi_{4}>\varphi_{1}>\varphi_{3}$ (see Figure 22).

Remark 2.6. As indicated in the title of [33, the inverse problem of the central configurations was studied in the $n$-body problem. Fix $q=\left(q_{1}, q_{2}, \cdots, q_{n}\right) \in\left(\mathbf{R}^{d}\right)^{n}$, and let $S(q)$ be the admissible set of masses by $S(q)=\left\{m=\left(m_{1}, m_{2}, \cdots, m_{n}\right) \mid m_{i}\right.$ $\in \mathbf{R}^{+}, q$ is a central configuration for $\left.m\right\}$. For a given $m \in S(q)$, let $S_{m}(q)$ be the permutational admissible set about $m=\left(m_{1}, m_{2}, \cdots, m_{n}\right)$ by

$$
S_{m}(q)=\left\{m^{\prime} \mid m^{\prime} \in S(q), m^{\prime} \neq m \text { and } m^{\prime} \text { is a permutation of } m\right\} .
$$

Configuration $q$ is called a super central configuration if there exists mass $m$ such that $S_{m}(q)$ is nonempty. The main results in 33 are the existence and classifications of super central configurations in the collinear four-body problem. For any fixed $q$ in the collinear four-body problem, one and only one of the following cases must apply: (1) $S_{m}(q)$ is an empty set; (2) $S_{m}(q)=\left\{\left(m_{4}, m_{3}, m_{2}, m_{1}\right)\right\}$; (3) $S_{m}(q)=\left\{\left(m_{2}, m_{4}, m_{1}, m_{3}\right)\right\} ;(4) S_{m}(q)=\left\{\left(m_{3}, m_{1}, m_{4}, m_{2}\right)\right\}$.

In this paper, we study the number of the geometric equivalence classes of the central configurations for any given positive four masses. The phenomenon of the decreased number of the geometric equivalence classes is closely related to the nonempty set $S_{m}(q)$ as stated in Theorem 1.7. 


\section{The Proof of Theorem 1.7}

Because central configuration is invariant up to translation and scaling, we can choose the coordinate system so that all four bodies are on the $x$-axis. Let $q_{1}=$ $-s-1, q_{2}=-1, q_{3}=1$, and $q_{4}=t+1$, where $s, t>0$. Then the set $W(n)$ can be modified to

$$
W(4)=\left\{q=(-s-1,-1,1, t+1) \in \mathbf{R}^{4} \mid s>0, t>0\right\} .
$$

Because we fix $q$ as the form $(-s-1,-1,1, t+1)$, we now allow $m_{i}$ s to change their order. When we say that $q=\left(q_{1}, q_{2}, q_{3}, q_{4}\right)$ is a collinear CC for $m(\alpha) \equiv$ $\left(m_{\alpha(1)}, m_{\alpha(2)}, m_{\alpha(3)}, m_{\alpha(4)}\right)$ with some $\alpha \in P(4)$, we always mean that $m_{\alpha(i)}$ is attached to $q_{i}$ for all $i=1,2,3,4$ and $q=(-s-1,-1,1, t+1)=(-s(\alpha)-$ $1,-1,1, t(\alpha)+1)$.

Proposition 3.1 (Geometric equivalence for collinear CCs in the four-body problem). For a given $m \in\left(\mathbf{R}^{+}\right)^{4}$, let $q$ and $p \in W(4)$ be two collinear CCs for $m(\alpha)$ and $m(\beta)$ with $\alpha$ and $\beta \in P(4)$, respectively. Then $q$ and $p$ are geometrically equivalent if and only if either $(s(\alpha), t(\alpha))=(s(\beta), t(\beta))$ or $(s(\alpha), t(\alpha))=(t(\beta), s(\beta))$.

Proof. Because $q$ and $p \in W(4), q_{2}=p_{2}=-1$ and $q_{3}=p_{3}=1$. By Definition 1.3, $q$ and $p$ are geometrically equivalent if and only if either $(-s(\alpha)-1,-1,1, t(\alpha)+1)=$ $a(-s(\beta)-1,-1,1, t(\beta)+1)$ or $(-s(\alpha)-1,-1,1, t(\alpha)+1)=a(t(\beta)+1,1,-1,-s(\beta)-$ $1)$. So $q$ and $p$ are geometrically equivalent if and only if either $(s(\alpha), t(\alpha))=$ $(s(\beta), t(\beta))$ or $(s(\alpha), t(\alpha))=(t(\beta), s(\beta))$.

Fix $m=\left(m_{1}, m_{2}, m_{3}, m_{4}\right) \in\left(\mathbf{R}^{+}\right)^{4}$. The central configuration equation (2) in collinear four bodies is

$$
A m=-\lambda q^{T}-u L^{T},
$$

where

$$
A=\left[\begin{array}{cccc}
0 & s^{-2} & (s+2)^{-2} & (s+t+2)^{-2} \\
-s^{-2} & 0 & 1 / 4 & (t+2)^{-2} \\
-(s+2)^{-2} & -1 / 4 & 0 & t^{-2} \\
-(s+t+2)^{-2} & -(t+2)^{-2} & -t^{-2} & 0
\end{array}\right],
$$

$u=\lambda c$ and $L=(1,1,1,1)$.

Because the matrix $A$ is skew symmetric, the determinant of $A$ is the square of its Pfaffian, that is, $\operatorname{det}(A)=(\operatorname{Pf} A)^{2}=\left[a_{12} a_{34}-a_{13} a_{24}+a_{14} a_{23}\right]^{2}=\left[(s t)^{-2}-\right.$ $\left.((s+2)(t+2))^{-2}+\frac{1}{4}(s+t+2)^{-2}\right]^{2}>0$. So the matrix has full rank. Therefore, the solution $m$ is uniquely determined by $s, t, \lambda, u$. Albouy and Moeckel 4 . proved that the given four-body collinear configuration determines a two-parameter family of masses making it central. Ouyang and Xie 22 found the unique solution of masses explicitly by standard row reduction, and we further simplify them to get

$$
\begin{array}{ll}
m_{1}=\frac{\lambda f_{1}-f_{2} u}{f_{5}}, & m_{2}=\frac{\lambda f_{3}+f_{4} u}{f_{5}}, \\
m_{3}=\frac{\lambda f_{33}-f_{44} u}{f_{5}}, & m_{4}=\frac{\lambda f_{11}+f_{22} u}{f_{5}},
\end{array}
$$


where $f_{i}, f_{j j}(i=1,2, \cdots, 5 ; j=1,2,3,4)$ are polynomials of $(s, t)$ defined in (6). On the other hand, F.R. Moulton proved that for a fixed mass vector $m=\left(m_{1}, m_{2}\right.$, $\left.\cdots, m_{n}\right)$ and a fixed order of the bodies along the line, there exists a unique collinear central configuration (up to translation and scaling [20]). So the configuration parameters $s, t, \lambda$ and $u$ are also uniquely determined by $m_{1}, m_{2}, m_{3}, m_{4}$. For each permutation of $m=\left(m_{1}, m_{2}, m_{3}, m_{4}\right)$, the corresponding $s, t$ produce exactly one collinear central configuration $q=\left(q_{1}, q_{2}, q_{3}, q_{4}\right)$. So we obtain ${ }^{\#} L(4, m) \leq 4 !=24$.

For any permutation $\tau \in P(4)$, we always denote the unique positive solution $s, t$ of (19) by $s(\tau), t(\tau)$ and the corresponding CC always by $q(\tau)=\left(q_{1}(\tau), q_{2}(\tau)\right.$, $\left.q_{3}(\tau), q_{4}(\tau)\right)=(-s(\tau)-1,-1,1, t(\tau)+1)$ for $m(\tau)=\left(m_{\tau(1)}, m_{\tau(2)}, m_{\tau(3)}, m_{\tau(4)}\right)$ so that the mass $m_{\tau(i)}$ is always put on the point $q_{i}(\tau)$ for $1 \leq i \leq 4$. Then we have

$$
\begin{array}{ll}
m_{\tau(1)}=\frac{\lambda(\tau) f_{1}-f_{2} u(\tau)}{f_{5}}, & m_{\tau(2)}=\frac{\lambda(\tau) f_{3}+f_{4} u(\tau)}{f_{5}}, \\
m_{\tau(3)}=\frac{\lambda(\tau) f_{33}-f_{44} u(\tau)}{f_{5}}, & m_{\tau(4)}=\frac{\lambda(\tau) f_{11}+f_{22} u(\tau)}{f_{5}},
\end{array}
$$

where $f_{i}=f_{i}(s(\tau), t(\tau)), f_{i i}=f_{i i}(s(\tau), t(\tau))$. Note that by Proposition 2.1, for two permutations $\alpha$ and $\beta$ in $P(4), q(\alpha) \sim q(\beta)$ if and only if either $s(\alpha)=s(\beta)$ and $t(\alpha)=t(\beta)$ or $s(\alpha)=t(\beta)$ and $t(\alpha)=s(\beta)$. We further prove some properties of equivalent central configurations.

Lemma 3.2. Given any $m \in\left(\mathbf{R}^{+}\right)^{4}$, let $q(\alpha)$ and $q(\beta)$ be CCs for $m(\alpha)$ and $m(\beta)$ with $\alpha, \beta \in P(4)$, respectively.

(i) If $q(\alpha) \sim q(\beta)$, then $\lambda(\alpha)=\lambda(\beta)$.

(ii) If $(s(\alpha), t(\alpha))=(s(\beta), t(\beta))$ and $m_{\alpha(i)}=m_{\beta(i)}$ for some $i \in\{1,2,3,4\}$, then $m(\alpha)=m(\beta)$.

(iii) If $(s(\alpha), t(\alpha))=(t(\beta), s(\beta))$ and $m_{\alpha(i)}=m_{\beta(5-i)}$ for some $i \in\{1,2,3,4\}$, then $m(\alpha)=m(\operatorname{con}(\beta))$.

Proof of Lemma 3.2. (i) Because the total mass $M=m_{1}+m_{2}+m_{3}+m_{4}$ is the same for $m(\alpha)$ and $m(\beta)$,

$$
M=m_{\alpha(1)}+m_{\alpha(2)}+m_{\alpha(3)}+m_{\alpha(4)}=m_{\beta(1)}+m_{\beta(2)}+m_{\beta(3)}+m_{\beta(4)} .
$$

From equations (22),

$$
\begin{aligned}
M & =\frac{\lambda(\alpha)\left(f_{1}+f_{3}+f_{11}+f_{33}\right)(s(\alpha), t(\alpha))-u(\alpha)\left(f_{2}-f_{4}+f_{44}-f_{22}\right)(s(\alpha), t(\alpha))}{f_{5}(s(\alpha), t(\alpha))} \\
& =\frac{\lambda(\beta)\left(f_{1}+f_{3}+f_{11}+f_{33}\right)(s(\beta), t(\beta))-u(\beta)\left(f_{2}-f_{4}+f_{44}-f_{22}\right)(s(\beta), t(\beta))}{f_{5}(s(\beta), t(\beta))} .
\end{aligned}
$$

By Proposition 2.1, $f_{2}-f_{4}+f_{44}-f_{22}=0$; then we have

$$
M=\frac{\lambda(\alpha)\left(f_{1}+f_{3}+f_{11}+f_{33}\right)(s(\alpha), t(\alpha))}{f_{5}(s(\alpha), t(\alpha))}=\frac{\lambda(\beta)\left(f_{1}+f_{3}+f_{11}+f_{33}\right)(s(\beta), t(\beta))}{f_{5}(s(\beta), t(\beta))} .
$$

Because $q(\alpha) \sim q(\beta), s(\alpha)=s(\beta)$ and $t(\alpha)=t(\beta)$ or $s(\alpha)=t(\beta)$ and $t(\alpha)=s(\beta)$. Then $\left(f_{1}+f_{3}+f_{11}+f_{33}\right)(s(\alpha), t(\alpha))=\left(f_{1}+f_{3}+f_{11}+f_{33}\right)(s(\beta), t(\beta))>0$ and $f_{5}(s(\alpha), t(\alpha))=f_{5}(s(\beta), t(\beta))$. We have $\lambda(\alpha)=\lambda(\beta)$.

(ii) If $m_{\alpha(i)}=m_{\beta(i)}$ for some $i$, and if $i=1$ we have

$$
m_{\alpha(1)}=\frac{\lambda(\alpha) f_{1}-f_{2} u(\alpha)}{f_{5}}=m_{\beta(1)}=\frac{\lambda(\beta) f_{1}-f_{2} u(\beta)}{f_{5}},
$$


which implies $u(\alpha)=u(\beta)$ because $f_{2}>0$ and $\lambda(\alpha)=\lambda(\beta)$ by $(i)$. Then $m(\alpha)=$ $m(\beta)$ because $(s, t, \lambda, u)(\alpha)=(s, t, \lambda, u)(\beta)$. Similarly it is true for $i=2,3,4$.

(iii) If $m_{\alpha(i)}=m_{\beta(5-i)}$ for some $i$, and if $i=1$ we have

$$
\begin{aligned}
& m_{\alpha(1)}=\frac{\lambda(\alpha) f_{1}(s(\alpha), t(\alpha))-f_{2}(s(\alpha), t(\alpha)) u(\alpha)}{f_{5}(s(\alpha), t(\alpha))} \\
= & m_{\beta(4)}=\frac{\lambda(\beta) f_{11}(s(\beta), t(\beta))+f_{22}(s(\beta), t(\beta)) u(\beta)}{f_{5}(s(\beta), t(\beta))} .
\end{aligned}
$$

Because $(s(\alpha), t(\alpha))=(t(\beta), s(\beta)), f_{1}(s(\alpha), t(\alpha))=f_{1}(t(\beta), s(\beta))=f_{11}(s(\beta), t(\beta))$ and $f_{2}(s(\alpha), t(\alpha))=f_{2}(t(\beta), s(\beta))=f_{22}(s(\beta), t(\beta))>0$, then $u(\alpha)=-u(\beta)$. So we have $(s(\alpha), t(\alpha), \lambda(\alpha), u(\alpha))=(t(\beta), s(\beta), \lambda(\beta),-u(\beta))$. Therefore $m(\alpha)=$ $m(\operatorname{con}(\beta))$ by (22).

So we complete the proof of Lemma 3.2

Claim 1. For permutations $\alpha=(1,2,3,4)$ and $\beta=(4,3,2,1), q(\alpha) \sim q(\beta)$.

Proof of Claim 1. In fact, for given $m(\alpha)$, we can implicitly solve for $s(\alpha), t(\alpha)$, $\lambda(\alpha)$ and $u(\alpha)$ which make $q(\alpha)$ central. Conversely, $m(\alpha)$ is uniquely determined by $s(\alpha), t(\alpha), \lambda(\alpha)$ and $u(\alpha)$ in equations (22). Note that the function of mass has a special symmetry in the following sense: $m_{1}(s, t, \lambda, u)=m_{4}(t, s, \lambda,-u)$ and $m_{2}(s, t, \lambda, u)=m_{3}(t, s, \lambda,-u)$. So $(-t(\alpha)-1,-1,1, s(\alpha)+1)$ is a central configuration for $m(\beta)$. By the uniqueness of solution for $m(\beta)$, we have $q(\beta)=$ $(-t(\alpha)-1,-1,1, s(\alpha)+1)$, which means $s(\alpha)=t(\beta)$ and $t(\alpha)=s(\beta)$. Thus $q(\alpha) \sim q(\beta)$.

Because of Claim 1, we obtain

$$
{ }^{\#} L(4, m) \leq \frac{4 !}{2}=12 .
$$

This can also be directly from the known inequality ${ }^{\#} L(4, m) \leq{ }^{\#} L_{P}(4, m)=\frac{4 !}{2}=$ 12. In order to know how many central configurations, we just need to discuss the geometric equivalence among the corresponding twelve permutations defined in (15) instead of the twenty-four permutations for each given mass $m=\left(m_{1}, m_{2}, m_{3}, m_{4}\right)$.

Next we study collinear CCs for different cases according to the different type $m$.

Case 1. Four equal masses: ${ }^{\#} L(4, m)={ }^{\#} L_{M}(4, m)=1$.

By equations (22) and the uniqueness of $s, t, \lambda, u$ for a given $m$, we have $q\left(\tau_{i}\right)=$ $q\left(\tau_{j}\right)$ for all $1 \leq i, j \leq 12$. In fact, the exact solution is $u=0, t=s$, and

$$
\lambda=\frac{160 s^{5}+656 s^{4}+1408 s^{3}+1664 s^{2}+1024 s+256+s^{8}+8 s^{7}+24 s^{6}}{4 m_{1}(s+2)^{2}(s+1)^{2} s^{2}\left(-4 s^{2}-16 s-16+s^{5}+5 s^{4}+8 s^{3}\right)},
$$

where $s$ is the unique positive zero of the polynomial equation

$$
s^{7}+7 s^{6}+19 s^{5}-84 s^{3}-152 s^{2}-112 s-32=0 .
$$

The numerical solution of (24) is $s=2.162120398$; then $\lambda=10.65968671 / m_{1}$.

So ${ }^{\#} L(4, m)={ }^{\#} L_{M}(4, m)=1$. 
Case 2. Three equal masses: ${ }^{\#} L(4, m)={ }^{\#} L_{M}(4, m)=2$.

Without loss of generality, we assume $m_{1}=m_{2}=m_{3}$, but $m_{3} \neq m_{4}$. By equations (22) and the uniqueness of $s, t, \lambda, u$ for a given $m$, we have either $q\left(\tau_{i}\right) \sim$ $q\left(\tau_{1}\right)$ or $q\left(\tau_{i}\right) \sim q\left(\tau_{2}\right)$ for all $1 \leq i \leq 12$. So we have either ${ }^{\#} L(4, m)=1$ if $q\left(\tau_{1}\right) \sim q\left(\tau_{2}\right)$ or $\# L(4, m)=2$ if $q\left(\tau_{1}\right)$ is not equivalent to $q\left(\tau_{2}\right)$.

If $q\left(\tau_{1}\right) \sim q\left(\tau_{2}\right)$, then $\lambda\left(\tau_{1}\right)=\lambda\left(\tau_{2}\right)$ by Lemma 3.2 If $s\left(\tau_{1}\right)=s\left(\tau_{2}\right)$ and $t\left(\tau_{1}\right)=$ $t\left(\tau_{2}\right)$, then $m\left(\tau_{1}\right)=m\left(\tau_{2}\right)$ because $m_{\tau_{1}(1)}=m_{\tau_{2}(1)}=m_{1}$. This implies $m_{3}=$ $m_{\tau_{1}(3)}=m_{\tau_{2}(3)}=m_{4}$, which contradicts $m_{3} \neq m_{4}$.

In addition, if $s\left(\tau_{1}\right)=t\left(\tau_{2}\right)$ and $t\left(\tau_{1}\right)=s\left(\tau_{2}\right)$, then by Lemma $3.2 m\left(\tau_{1}\right)=$ $m\left(\operatorname{con}\left(\tau_{2}\right)\right)$ because $m_{\tau_{1}(1)}=m_{\tau_{2}(4)}$. Therefore $m_{2}=m_{\tau_{1}(2)}=m_{\tau_{2}(3)}=m_{4}$, which is a contradiction. So $q\left(\tau_{1}\right) \sim q\left(\tau_{2}\right)$ is impossible. We proved that ${ }^{\#} L(4, m)=\#$ $L_{M}(4, m)=2$ for three equal masses.

Case 3. Two pair of equal masses: ${ }^{\#} L(4, m)={ }^{\#} L_{M}(4, m)=4$.

Without loss of generality, we assume $m_{1}=m_{4}, m_{2}=m_{3}$, but $m_{1} \neq m_{2}$. Then the possible nonequivalent central configurations are corresponding to the permutations $\tau_{1}, \tau_{2}, \tau_{5}$, and $\tau_{8}$. Using Lemma 3.2, we show that none of any two are equivalent by contradiction.

(1) In fact, if $q\left(\tau_{1}\right) \sim q\left(\tau_{2}\right)$, then we have $m\left(\tau_{1}\right)=m\left(\tau_{2}\right)$ or $m\left(\tau_{1}\right)=m\left(\operatorname{con}\left(\tau_{2}\right)\right)$ because $m_{\tau_{1}(1)}=m_{\tau_{2}(1)}$ and $m_{\tau_{1}(4)}=m_{\tau_{2}(5-4)}=m_{\tau_{2}(1)}$. This contradicts $m_{1} \neq$ $m_{2}$. Therefore $q\left(\tau_{1}\right)$ is not equivalent to $q\left(\tau_{2}\right)$. By the same arguments, we have the following:

(2) Because $m_{\tau_{1}(1)}=m_{\tau_{5}(1)}$ and $m_{\tau_{1}(2)}=m_{\tau_{5}(5-2)}=m_{\tau_{5}(3)}, q\left(\tau_{1}\right) \nsim q\left(\tau_{5}\right)$.

(3) Because $m_{\tau_{2}(1)}=m_{\tau_{5}(1)}$ and $m_{\tau_{2}(2)}=m_{\tau_{5}(5-2)}=m_{\tau_{5}(3)}, q\left(\tau_{2}\right) \nsim q\left(\tau_{5}\right)$.

(4) Because $m_{\tau_{2}(4)}=m_{\tau_{8}(4)}$ and $m_{\tau_{2}(3)}=m_{\tau_{8}(5-3)}=m_{\tau_{8}(2)}, q\left(\tau_{2}\right) \nsim q\left(\tau_{8}\right)$.

(5) Because $m_{\tau_{5}(4)}=m_{\tau_{8}(3)}$ and $m_{\tau_{5}(2)}=m_{\tau_{8}(5-2)}=m_{\tau_{8}(3)}, q\left(\tau_{5}\right) \nsim q\left(\tau_{8}\right)$.

(6) By the unique corresponding between the masses and $(s, t, \lambda, u)$ and the symmetry of masses with respect to positions, we have $s\left(\tau_{1}\right)=t\left(\tau_{1}\right)$ and $u\left(\tau_{1}\right)=0$, and $s\left(\tau_{8}\right)=t\left(\tau_{8}\right)$ and $u\left(\tau_{8}\right)=0$. If $q\left(\tau_{1}\right) \sim q\left(\tau_{8}\right)$, then $s\left(\tau_{1}\right)=t\left(\tau_{1}\right)=s\left(\tau_{8}\right)=t\left(\tau_{8}\right)$ and $\lambda\left(\tau_{1}\right)=\lambda\left(\tau_{8}\right)$. Then $m\left(\tau_{1}\right)=m\left(\tau_{8}\right)$, which contradicts $m_{1} \neq m_{2}$. So $q\left(\tau_{1}\right) \not$ $q\left(\tau_{8}\right)$.

We have proved that ${ }^{\#} L(4, m)={ }^{\#} L_{M}(4, m)=4$ if two pair of masses are equal.

Case 4. Only one pair of equal masses: ${ }^{\#} L(4, m)={ }^{\#} L_{M}(4, m)=6$.

Without loss of generality, we assume $m_{1}=m_{4}, m_{2}, m_{3}$, and $m_{4}$ are mutually distinct. The possible nonequivalent central configurations are corresponding to the permutations $\tau_{1}, \tau_{2}, \tau_{4}, \tau_{5}, \tau_{6}, \tau_{8}$. To show that none of any two central configurations among them are equivalent, we need to discuss the $\left(\begin{array}{l}6 \\ 2\end{array}\right)=15$ cases. However, we only need to check the following three cases: $q\left(\tau_{1}\right) \nsim q\left(\tau_{8}\right), q\left(\tau_{2}\right) \nsim q\left(\tau_{4}\right)$, and $q\left(\tau_{5}\right) \nsim q\left(\tau_{6}\right)$ after we use Lemma 3.2 to other cases with the same arguments as shown in Case $3(2)$.

(1) $q\left(\tau_{1}\right) \nsim q\left(\tau_{8}\right)$.

(a) If $q\left(\tau_{1}\right) \sim q\left(\tau_{8}\right)$ and $\left(s\left(\tau_{1}\right), t\left(\tau_{1}\right)\right)=\left(s\left(\tau_{8}\right), t\left(\tau_{8}\right)\right)$, then $\lambda\left(\tau_{1}\right)=\lambda\left(\tau_{8}\right)$, $f_{i}\left(s\left(\tau_{1}\right), t\left(\tau_{1}\right)\right)=f_{i}\left(s\left(\tau_{8}\right), t\left(\tau_{8}\right)\right)$ and $f_{i i}\left(s\left(\tau_{1}\right), t\left(\tau_{1}\right)\right)=f_{i i}\left(s\left(\tau_{8}\right), t\left(\tau_{8}\right)\right)$. By equation (22) and $m_{\tau_{1}(1)}=m_{\tau_{8}(2)}=m_{1}$ and $m_{\tau_{1}(2)}=m_{\tau_{8}(1)}=m_{2}$, we have

$$
\lambda\left(\tau_{1}\right) f_{1}-f_{2} u\left(\tau_{1}\right)=\lambda\left(\tau_{8}\right) f_{3}+f_{4} u\left(\tau_{8}\right), \quad \lambda\left(\tau_{1}\right) f_{3}+f_{4} u\left(\tau_{1}\right)=\lambda\left(\tau_{8}\right) f_{1}-f_{2} u\left(\tau_{8}\right) .
$$


Adding the two equations and rearranging the terms, we have

$$
\left(-f_{2}+f_{4}\right) u\left(\tau_{1}\right)=\left(-f_{2}+f_{4}\right) u\left(\tau_{8}\right),
$$

which implies that $u\left(\tau_{1}\right)=u\left(\tau_{8}\right)$ because $f_{2}>f_{4}>0$ for any $s>0, t>0$. Then $m\left(\tau_{1}\right)=m\left(\tau_{8}\right)$. It contradicts $m\left(\tau_{1}\right) \neq m\left(\tau_{8}\right)$.

(b) If $q\left(\tau_{1}\right) \sim q\left(\tau_{8}\right)$ and $\left(s\left(\tau_{1}\right), t\left(\tau_{1}\right)\right)=\left(t\left(\tau_{8}\right), s\left(\tau_{8}\right)\right)$, then $\lambda\left(\tau_{1}\right)=\lambda\left(\tau_{8}\right)$, $f_{i}\left(s\left(\tau_{1}\right), t\left(\tau_{1}\right)\right)=f_{i i}\left(s\left(\tau_{8}\right), t\left(\tau_{8}\right)\right)$. Using the fact that $m_{\tau_{1}(1)}=m_{\tau_{8}(2)}=m_{1}$ and $m_{\tau_{1}(2)}=m_{\tau_{8}(1)}=m_{2}$, we solve for $u\left(\tau_{1}\right)$ and $u\left(\tau_{8}\right)$,

$$
u\left(\tau_{1}\right)=\frac{\lambda g_{81}}{-f_{44} f_{4}+f_{22} f_{2}}, \quad u\left(\tau_{8}\right)=-\frac{\lambda g_{82}}{-f_{44} f_{4}+f_{22} f_{2}},
$$

where $g_{81}=\left(f_{44} f_{3}-f_{22} f_{33}-f_{44} f_{11}+f_{22} f_{1}\right), g_{82}=\left(f_{1} f_{4}+f_{2} f_{3}-f_{2} f_{11}\right.$ $\left.-f_{33} f_{4}\right)$ and $f_{i}=f_{i}\left(s\left(\tau_{1}\right), t\left(\tau_{1}\right)\right), f_{i i}=f_{i i}\left(s\left(\tau_{1}\right), t\left(\tau_{1}\right)\right)$. Because

$$
m_{\tau_{1}(3)}-m_{\tau_{8}(4)}=\frac{\lambda\left(f_{2}+f_{44}\right)\left(g_{81}-g_{82}\right)}{\left(-f_{44} f_{4}+f_{22} f_{2}\right) f_{5}},
$$

$m_{\tau_{1}(3)}=m_{\tau_{8}(4)}=m_{3}$ is equivalent to $g_{81}=g_{82}$, which implies $u\left(\tau_{1}\right)=-u\left(\tau_{8}\right)$. Therefore $m\left(\tau_{1}\right)=m\left(\operatorname{con}\left(\tau_{8}\right)\right)$. It contradicts $m\left(\tau_{1}\right) \neq m\left(\operatorname{con}\left(\tau_{8}\right)\right)$.

These two contradictions prove that $q\left(\tau_{1}\right) \nsim q\left(\tau_{8}\right)$.

(2) $q\left(\tau_{2}\right) \nsim q\left(\tau_{4}\right)$.

(a) If $q\left(\tau_{2}\right) \sim q\left(\tau_{4}\right)$ and $\left(s\left(\tau_{2}\right), t\left(\tau_{2}\right)\right)=\left(s\left(\tau_{4}\right), t\left(\tau_{4}\right)\right)$, then $m\left(\tau_{2}\right)=m\left(\tau_{4}\right)$ because $m_{\tau_{2}(1)}=m_{\tau_{4}(1)}=m_{1}$. It contradicts $m\left(\tau_{2}\right) \neq m\left(\tau_{4}\right)$.

(b) If $q\left(\tau_{2}\right) \sim q\left(\tau_{4}\right)$ and $\left(s\left(\tau_{2}\right), t\left(\tau_{2}\right)\right)=\left(t\left(\tau_{4}\right), s\left(\tau_{4}\right)\right)$, then $\lambda\left(\tau_{2}\right)=\lambda\left(\tau_{4}\right)$, $f_{i}\left(s\left(\tau_{2}\right), t\left(\tau_{2}\right)\right)=f_{i i}\left(s\left(\tau_{4}\right), t\left(\tau_{4}\right)\right)$. Using the fact that $m_{\tau_{2}(1)}=m_{\tau_{4}(1)}=m_{1}$ and $m_{\tau_{2}(2)}=m_{\tau_{4}(4)}=m_{2}$, we solve for $u\left(\tau_{2}\right)$ and $u\left(\tau_{4}\right)$,

$$
\begin{aligned}
& u\left(\tau_{2}\right)=\frac{\lambda\left(f_{2} f_{1}-f_{22} f_{1}-f_{2} f_{11}+f_{22} f_{3}\right)}{f_{2}{ }^{2}-f_{22} f_{4}}, \\
& u\left(\tau_{4}\right)=-\frac{\lambda\left(-f_{3} f_{2}-f_{4} f_{1}+f_{4} f_{11}+f_{2} f_{1}\right)}{f_{2}{ }^{2}-f_{22} f_{4}} .
\end{aligned}
$$

Substituting $u\left(\tau_{2}\right)$ into equation (22), we have

$$
\begin{gathered}
m_{\tau_{2}(1)}=\frac{\lambda\left(f_{1} f_{22} f_{4}-f_{2} f_{22} f_{1}-f_{2}{ }^{2} f_{11}+f_{2} f_{22} f_{3}\right)}{\left(-f_{2}{ }^{2}+f_{22} f_{4}\right) f_{5}}, \\
m_{\tau_{2}(2)}=-\frac{\lambda\left(f_{3} f_{2}{ }^{2}+f_{4} f_{2} f_{1}-f_{1} f_{22} f_{4}-f_{4} f_{2} f_{11}\right)}{\left(-f_{2}{ }^{2}+f_{22} f_{4}\right) f_{5}}, \\
m_{\tau_{2}(3)}=\frac{\lambda\left(-f_{33} f_{2}{ }^{2}+f_{33} f_{22} f_{4}+f_{44} f_{2} f_{1}-f_{44} f_{22} f_{1}-f_{44} f_{2} f_{11}+f_{44} f_{22} f_{3}\right)}{\left(-f_{2}{ }^{2}+f_{22} f_{4}\right) f_{5}} \\
m_{\tau_{2}(4)}=\frac{\lambda\left(-f_{2}{ }^{2} f_{11}+f_{11} f_{22} f_{4}-f_{2} f_{22} f_{1}+f_{22}{ }^{2} f_{1}+f_{22} f_{2} f_{11}-f_{22}{ }^{2} f_{3}\right)}{\left(-f_{2}{ }^{2}+f_{22} f_{4}\right) f_{5}}, \\
m_{\tau_{2}(3)-} m_{\tau_{4}(3)}=\frac{\lambda g_{51}}{\left(-f_{2}{ }^{2}+f_{22} f_{4}\right) f_{5}} \text { and } m_{\tau_{2}(4)}-m_{\tau_{4}(2)}=\frac{\lambda g_{51}}{\left(-f_{2}{ }^{2}+f_{22} f_{4}\right) f_{5}}, \text { where } \\
g_{51}=f_{33} f_{2}{ }^{2}-f_{33} f_{22} f_{4}-f_{44} f_{2} f_{1}+f_{44} f_{22} f_{1}+f_{44} f_{2} f_{11} \\
-f_{44} f_{22} f_{3}-f_{3} f_{2}{ }^{2}+f_{3} f_{22} f_{4}+f_{4} f_{3} f_{2}+f_{4}{ }^{2} f_{1}-f_{4}{ }^{2} f_{11}-f_{4} f_{2} f_{1}
\end{gathered}
$$

and $m_{\tau_{2}(3)}=m_{\tau_{4}(3)}$ and $m_{\tau_{2}(4)}=m_{\tau_{4}(2)}$ are equivalent to $g_{51}=0$. However, $m_{\tau_{2}(1)}$ is always nonpositive along the implicit curve $\Gamma_{51}$ on which $g_{51}=0$ from the following proposition (see Figure 3). The proof of the proposition is easily adapted from the proof of proposition 5.3 in 33 . This proves that $q\left(\tau_{2}\right) \nsim q\left(\tau_{4}\right)$. 


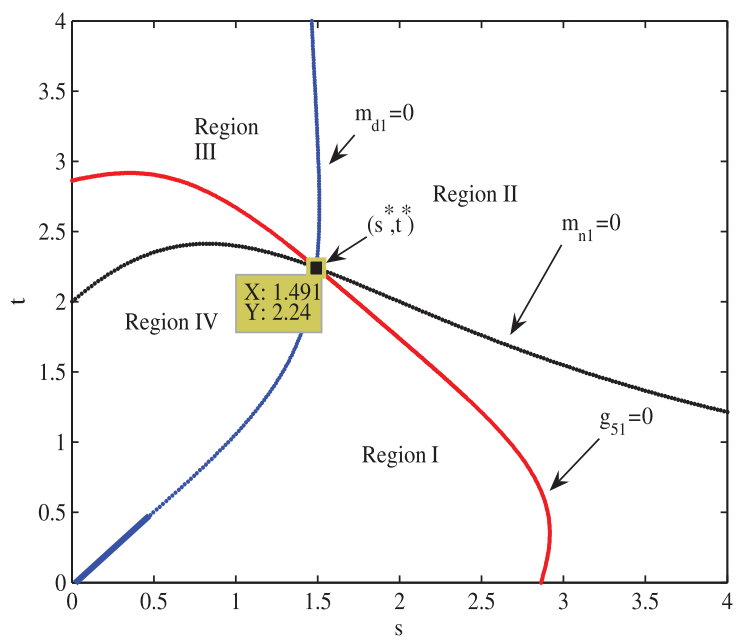

Figure 3. Implicit curve $\Gamma_{51}$ is in Region I and Region III. $m_{\tau_{2}(1)}$ given by (27) in case (4) is negative along $\Gamma_{51}$.

Proposition 3.3. Let $_{g_{p 51}}$ be the polynomial such that $g_{51}(s, t)=-4 s^{2} t^{2}(s-t)(s+$ $t+2)^{2} g_{p 51}$ and let

$$
\Gamma_{51}=\left\{(s, t) \in\left(\mathbf{R}^{+}\right)^{2} \mid g_{p 51}(s, t)=0\right\} .
$$

(i) $g_{51}(s, t)=-g_{51}(t, s)$ holds for all $(s, t) \in\left(\mathbf{R}^{+}\right)^{2}$. Then the points on $g_{51}(s, t)=0$ are symmetric about $s=t$ and $g_{51}(s, s)=0$ for all $s>0$.

(ii) $m_{\tau_{2}(1)}$ given by (27) in case (4) is nonpositive along $\Gamma_{51}$.

(3) $q\left(\tau_{5}\right) \nsim q\left(\tau_{6}\right)$.

(a) If $q\left(\tau_{5}\right) \sim q\left(\tau_{6}\right)$ and $\left(s\left(\tau_{5}\right), t\left(\tau_{5}\right)\right)=\left(s\left(\tau_{6}\right), t\left(\tau_{6}\right)\right)$, then $m\left(\tau_{5}\right)=m\left(\tau_{6}\right)$ because $m_{\tau_{5}(1)}=m_{\tau_{6}(1)}=m_{1}$. It contradicts $m\left(\tau_{5}\right) \neq m\left(\tau_{6}\right)$.

(b) If $q\left(\tau_{5}\right) \sim q\left(\tau_{6}\right)$ and $\left(s\left(\tau_{5}\right), t\left(\tau_{5}\right)\right)=\left(t\left(\tau_{6}\right), s\left(\tau_{6}\right)\right)$, then $\lambda\left(\tau_{5}\right)=\lambda\left(\tau_{6}\right)$, $f_{i}\left(s\left(\tau_{5}\right), t\left(\tau_{5}\right)\right)=f_{i i}\left(s\left(\tau_{6}\right), t\left(\tau_{6}\right)\right)$. Using the fact that $m_{\tau_{5}(1)}=m_{\tau_{6}(1)}=m_{1}$ and $m_{\tau_{5}(2)}=m_{\tau_{6}(2)}=m_{4}$, we solve for $u\left(\tau_{5}\right)$ and $u\left(\tau_{6}\right)$,

$$
\begin{aligned}
& u\left(\tau_{5}\right)=\frac{\lambda\left(f_{1} f_{44}-f_{11} f_{44}+f_{22} f_{3}-f_{22} f_{33}\right)}{f_{2} f_{44}-f_{4} f_{22}}, \\
& u\left(\tau_{6}\right)=\frac{\lambda\left(f_{2} f_{3}-f_{2} f_{33}+f_{4} f_{1}-f_{4} f_{11}\right)}{f_{2} f_{44}-f_{4} f_{22}} .
\end{aligned}
$$

$m_{\tau_{5}(3)}-m_{\tau_{6}(4)}=-\frac{\lambda g_{41}}{\left(f_{2} f_{44}-f_{4} f_{22}\right) f_{5}}$, where

$$
\begin{aligned}
& g_{41}=-f_{33} f_{2} f_{44}+f_{4} f_{22} f_{33}+f_{1} f_{44}{ }^{2}-f_{11} f_{44}{ }^{2}+f_{44} f_{22} f_{3}-f_{44} f_{22} f_{33} \\
& +f_{1} f_{2} f_{44}-f_{1} f_{4} f_{22}+f_{2}{ }^{2} f_{3}-f_{2}{ }^{2} f_{33}+f_{2} f_{4} f_{1}-f_{2} f_{4} f_{11} .
\end{aligned}
$$

$m_{\tau_{5}(3)}=m_{\tau_{6}(4)}$ is equivalent to $g_{41}=0$. By direct computation $g_{41}=(s-t) g_{411}$, where $g_{411}$ is a polynomial with all positive terms. So $t$ must be equal to $s$ if $g_{41}=0$. But when $t=s, m\left(\tau_{5}\right)=m\left(\tau_{6}\right)$ because $\left(s\left(\tau_{5}\right), t\left(\tau_{5}\right)\right)=\left(s\left(\tau_{6}\right), t\left(\tau_{6}\right)\right)=(s, s)$. It contradicts $m\left(\tau_{5}\right) \neq m\left(\tau_{6}\right)$.

These two contradictions prove that $q\left(\tau_{5}\right) \nsim q\left(\tau_{6}\right)$.

We have proved that ${ }^{\#} L(4, m)={ }^{\#} L_{M}(4, m)=6$ if only one pair of masses are equal. 
Case 5 and Case 6. Four mutually distinct masses: ${ }^{\#} L(4, m)=11$ if $m \in H_{4}$, and ${ }^{\#} L(4, m)=12$ if $m \notin H_{4}$.

To prove Case 5 and Case 6 , we first show the following lemmas. The proofs of the lemmas are given in section 4 .

Lemma 3.4. If $m_{1}, m_{2}, m_{3}, m_{4}$ are mutually distinct positive masses, then it is impossible that $q(\theta) \sim q(\alpha) \sim q(\beta)$ for mutually distinct $\theta, \alpha$, and $\beta \in\left\{\tau_{i} \mid i=\right.$ $1, \cdots, 12\}$.

Lemma 3.5 (Nonequivalency). If $m_{1}, m_{2}, m_{3}, m_{4}$ are mutually distinct positive masses, then

(1) $q\left(\tau_{1}\right) \nsim q\left(\tau_{i}\right)$ for $i \in\{1,2, \cdots, 12\} \backslash\{1,10\}$;

(2) $q\left(\tau_{2}\right) \not q q\left(\tau_{i}\right)$ for $i \in\{1,2, \cdots, 12\} \backslash\{2,9\}$;

(3) $q\left(\tau_{3}\right) \not q\left(\tau_{i}\right)$ for $i \in\{1,2, \cdots, 12\} \backslash\{3,8\}$;

(4) $q\left(\tau_{4}\right) \nsim q\left(\tau_{i}\right)$ for $i \in\{1,2, \cdots, 12\} \backslash\{4,12\}$;

(5) $q\left(\tau_{5}\right) \not q\left(\tau_{i}\right)$ for $i \in\{1,2, \cdots, 12\} \backslash\{5,7\}$;

(6) $q\left(\tau_{6}\right) \not q\left(\tau_{i}\right)$ for $i \in\{1,2, \cdots, 12\} \backslash\{6,11\}$.

Lemma 3.6 (Equivalency). Let $\Psi_{i}$ be the singular surfaces in the mass space which is defined in Definition (2.3). If $m_{1}, m_{2}, m_{3}, m_{4}$ are mutually distinct positive masses, then

(1) $q\left(\tau_{1}\right) \sim q\left(\tau_{10}\right)$ if and only if $m=\left(m_{1}, m_{2}, m_{3}, m_{4}\right) \in \Psi_{1}$;

(2) $q\left(\tau_{2}\right) \sim q\left(\tau_{9}\right)$ if and only if $m=\left(m_{1}, m_{2}, m_{3}, m_{4}\right) \in \Psi_{2}$;

(3) $q\left(\tau_{3}\right) \sim q\left(\tau_{8}\right)$ if and only if $m=\left(m_{1}, m_{2}, m_{3}, m_{4}\right) \in \Psi_{3}$;

(4) $q\left(\tau_{4}\right) \sim q\left(\tau_{12}\right)$ if and only if $m=\left(m_{1}, m_{2}, m_{3}, m_{4}\right) \in \Psi_{4}$;

(5) $q\left(\tau_{5}\right) \sim q\left(\tau_{7}\right)$ if and only if $m=\left(m_{1}, m_{2}, m_{3}, m_{4}\right) \in \Psi_{5}$;

(6) $q\left(\tau_{6}\right) \sim q\left(\tau_{11}\right)$ if and only if $m=\left(m_{1}, m_{2}, m_{3}, m_{4}\right) \in \Psi_{6}$.

Lemma 3.7. If $m_{1}, m_{2}, m_{3}, m_{4}$ are mutually distinct positive masses, then it is impossible that $q\left(\tau_{i}\right) \sim q\left(\tau_{j}\right)$ and $q\left(\tau_{k}\right) \sim q\left(\tau_{l}\right)$ hold simultaneously for $1 \leq i, j, k, l \leq$ 12 , and $i, j, k, l$ are mutually distinct.

By Lemma 3.4 and Lemma 3.7, we have either ${ }^{\#} L(4, m)=11$ or ${ }^{\#} L(4, m)=12$ for any given four distinct positive masses. By Lemma 3.6, we further know that ${ }^{\#} L(4, m)=11$ if $m \in H_{4}$ and ${ }^{\#} L(4, m)=12$ if $m \notin H_{4}$ for any given four mutually distinct positive masses.

\section{Proofs of Lemma 3.4 Lemma 3.7}

Proof of Lemma 3.4. The idea of Lemma 3.4 and its proof are from the work of Z.Xie [33. We prove it by contradiction. We assume that $q(\theta) \sim q(\alpha) \sim q(\beta)$ for some mutually distinct $\theta, \alpha$, and $\beta \in\left\{\tau_{i} \mid i=1, \cdots, 12\right\}$. Then $q(\theta) \sim q(\alpha) \sim q(\beta) \sim$ $q(\operatorname{con}(\theta)) \sim q(\operatorname{con}(\alpha)) \sim q(\operatorname{con}(\beta))$. We have either $(s(\theta), t(\theta))=(s(\alpha), t(\alpha))$ or $(s(\theta), t(\theta))=(t(\alpha), s(\alpha))$. So we can assume that $(s(\theta), t(\theta))=(s(\alpha), t(\alpha))=$ $(s(\beta), t(\beta))$ because $(s(\theta), t(\theta))=(t(\operatorname{con}(\theta)), s(\operatorname{con}(\theta)))$.

By Lemma 3.2 we have

$$
\lambda(\theta)=\lambda(\alpha)=\lambda(\beta) .
$$

Without loss of generality, we can assume $u(\alpha)<u(\beta)$. So $u(\theta), u(\alpha)$ and $u(\beta)$ give us three distinct solutions $m(\theta), m(\alpha)$ and $m(\beta)$ through equation (22) for the same $s, t, \lambda$. Note that $m_{\alpha(i)} i=1,2,3,4$, are linear functions of $u(\alpha)$. Because $f_{2}>0$, $f_{4}>0, f_{22}>0$, and $f_{44}>0$ for all $s>0, t>0, m_{\alpha(1)}, m_{\alpha(3)}$ are decreasing with 


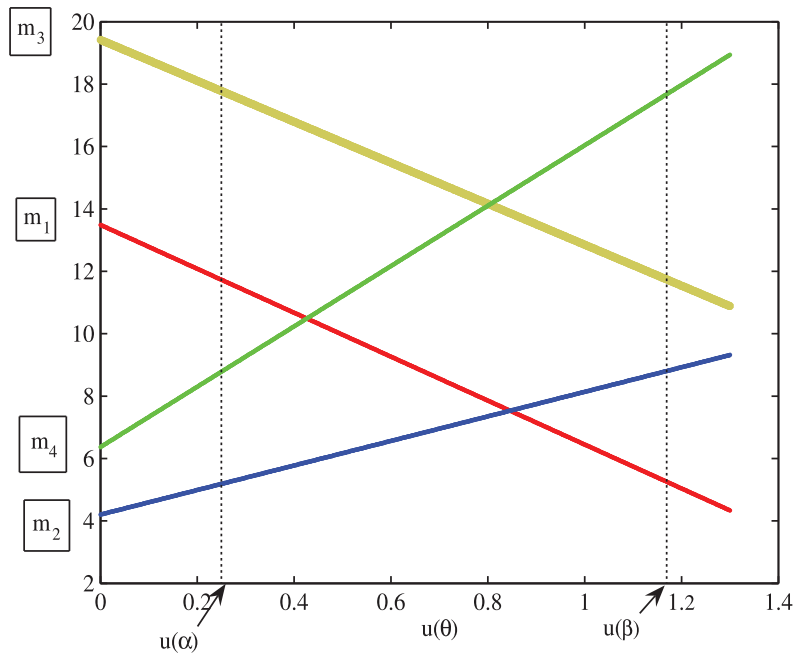

Figure 4. The graph $m_{i}$ of (22) for fixed $(s, t)=$ $(1.8,2.542561504)$ and $\lambda=1$. It is impossible to have three distinct $u(\theta), u(\alpha), u(\beta)$ such that $q(\theta) \sim q(\alpha) \sim q(\beta)$.

respect to $u(\alpha)$ and $m_{\alpha(2)}, m_{\alpha(4)}$ are increasing with respect to $u(\alpha)$ (an example as shown in Figure (4). Then $\max \left\{m_{\alpha(i)}, i=1,2,3,4\right\}$ must be either $m_{\alpha(1)}$ or $m_{\alpha(3)}$, i.e.

$$
\max \left\{m_{\alpha(i)}, i=1,2,3,4\right\}=\max \left\{m_{\alpha(i)}, i=1,3\right\} .
$$

If not, i.e. $\max \left\{m_{\alpha(i)}, i=1,2,3,4\right\}$ is either $m_{\alpha(2)}$ or $m_{\alpha(4)}$, then

$$
\max \left\{m_{\beta(i)}, i=2,4\right\}>\max \left\{m_{\alpha(i)}, i=2,4\right\}=\max \left\{m_{\alpha(i)}, i=1,2,3,4\right\}
$$

because $u(\beta)>u(\alpha)$. Then

$$
\max \left\{m_{\beta(i)}, i=1,2,3,4\right\} \geq \max \left\{m_{\beta(i)}, i=2,4\right\}>\max \left\{m_{\alpha(i)}, i=1,2,3,4\right\},
$$

which contradicts $\left\{m_{\beta(i)}, i=1,2,3,4\right\}=\left\{m_{\alpha(i)}, i=1,2,3,4\right\}$. The contradiction confirms that

$$
\max \left\{m_{\alpha(i)}, i=1,2,3,4\right\}=\max \left\{m_{\alpha(1)}, m_{\alpha(3)}\right\} .
$$

Similar arguments imply

$$
\max \left\{m_{\beta(i)}, i=1,2,3,4\right\}=\max \left\{m_{\beta(2)}, m_{\beta(4)}\right\} .
$$

Because $u(\theta), u(\alpha), u(\beta)$ are distinct, then one of the following cases must apply: (a) $u(\theta)<u(\alpha)$, (b) $u(\alpha)<u(\theta)<u(\beta)$, (c) $u(\beta)<u(\theta)$. However, none of the three cases is true.

In fact, suppose (a) $u(\theta)<u(\alpha)$ is true. Then

$$
\begin{gathered}
\max \left\{m_{\theta(i)}, i=1,2,3,4\right\} \geq \max \left\{m_{\theta(1)}, m_{\theta(3)}\right\} \\
>\max \left\{m_{\alpha(1)}, m_{\alpha(3)}\right\}=\max \left\{m_{\alpha(i)}, i=1,2,3,4\right\},
\end{gathered}
$$

which contradicts $\left\{m_{\theta(i)}, i=1,2,3,4\right\}=\left\{m_{\alpha(i)}, i=1,2,3,4\right\}$.

Suppose (b) $u(\alpha)<u(\theta)<u(\beta)$ is true. 
There are two cases. If $\max \left\{m_{\theta(i)}, i=1,2,3,4\right\}=\max \left\{m_{\theta(1)}, m_{\theta(3)}\right\}$,

$$
\max \left\{m_{\alpha(1)}, m_{\alpha(3)}\right\}=\max \left\{m_{\alpha(i)}, i=1,2,3,4\right\}>\max \left\{m_{\theta(i)}, i=1,2,3,4\right\},
$$

and if $\max \left\{m_{\theta(i)}, i=1,2,3,4\right\}=\max \left\{m_{\theta(2)}, m_{\theta(4)}\right\}$, then

$$
\max \left\{m_{\beta(2)}, m_{\beta(4)}\right\}=\max \left\{m_{\beta(i)}, i=1,2,3,4\right\}>\max \left\{m_{\theta(i)}, i=1,2,3,4\right\} .
$$

Both of them contradict

$$
\left\{m_{\theta(i)}, i=1,2,3,4\right\}=\left\{m_{\alpha(i)}, i=1,2,3,4\right\}=\left\{m_{\beta(i)}, i=1,2,3,4\right\} .
$$

Suppose (c) $u(\beta)<u(\theta)$; then

$$
\begin{gathered}
\max \left\{m_{\beta(i)}, i=1,2,3,4\right\}=\max \left\{m_{\beta(2)}, m_{\beta(4)}\right\} \\
<\max \left\{m_{\theta(2)}, m_{\theta(4)}\right\} \leq \max \left\{m_{\theta(i)}, i=1,2,3,4\right\},
\end{gathered}
$$

which contradicts $\left\{m_{\theta(i)}, i=1,2,3,4\right\}=\left\{m_{\beta(i)}, i=1,2,3,4\right\}$.

The contradictions prove that it is impossible that $q(\theta) \sim q(\alpha) \sim q(\beta)$ for mutually distinct $\theta, \alpha$, and $\beta \in\left\{\tau_{i} \mid i=1, \cdots, 12\right\}$.

Proof of Lemma 3.5. If $m_{1}, m_{2}, m_{3}, m_{4}$ are four mutually distinct positive masses, then $m\left(\tau_{i}\right) \neq m\left(\tau_{j}\right)$ and $m\left(\tau_{i}\right) \neq m\left(\operatorname{con}\left(\tau_{j}\right)\right)$ for any $1 \leq i \neq j \leq 12$. We first prove that $q\left(\tau_{1}\right) \nsim q\left(\tau_{i}\right)$ for $i \in\{1,2, \cdots, 12\} \backslash\{1,10\}$.

(a) $q\left(\tau_{1}\right) \nsim q\left(\tau_{2}\right)$.

$\left(a_{1}\right)$ If $q\left(\tau_{1}\right) \sim q\left(\tau_{2}\right)$ and $\left(s\left(\tau_{1}\right), t\left(\tau_{1}\right)\right)=\left(s\left(\tau_{2}\right), t\left(\tau_{2}\right)\right)$, then $m\left(\tau_{1}\right)=m\left(\tau_{2}\right)$ because $m_{\tau_{1}(1)}=m_{\tau_{2}(1)}$ by Lemma 3.2. This contradicts $m\left(\tau_{1}\right) \neq m\left(\tau_{2}\right)$.

$\left(a_{2}\right)$ If $q\left(\tau_{1}\right) \sim q\left(\tau_{2}\right)$ and $\left(s\left(\tau_{1}\right), t\left(\tau_{1}\right)\right)=\left(t\left(\tau_{2}\right), s\left(\tau_{2}\right)\right)$, then $\lambda\left(\tau_{1}\right)=\lambda\left(\tau_{2}\right)$, $f_{i}\left(s\left(\tau_{1}\right), t\left(\tau_{1}\right)\right)=f_{i i}\left(s\left(\tau_{2}\right), t\left(\tau_{2}\right)\right)$. Using the fact that $m_{\tau_{1}(1)}=m_{\tau_{2}(1)}=m_{1}$ and $m_{\tau_{1}(2)}=m_{\tau_{2}(2)}=m_{2}$, we solve for $u\left(\tau_{1}\right)$ and $u\left(\tau_{2}\right)$,

$$
\begin{gathered}
u\left(\tau_{1}\right)=\frac{\lambda\left(f_{1} f_{44}-f_{11} f_{44}+f_{22} f_{3}-f_{22} f_{33}\right)}{f_{2} f_{44}-f_{4} f_{22}}, \\
u\left(\tau_{2}\right)=\frac{\lambda\left(f_{2} f_{3}-f_{2} f_{33}+f_{4} f_{1}-f_{4} f_{11}\right)}{f_{2} f_{44}-f_{4} f_{22}} . \\
m_{\tau_{1}(3)}-m_{\tau_{2}(4)}=-\frac{\lambda g_{12}}{\left(f_{2} f_{44}-f_{4} f_{22}\right) f_{5}}, \text { where } \\
g_{12}=-f_{33} f_{2} f_{44}+f_{4} f_{22} f_{33}+f_{1} f_{44}{ }^{2}-f_{11} f_{44}{ }^{2}+f_{44} f_{22} f_{3}-f_{44} f_{22} f_{33} \\
+f_{1} f_{2} f_{44}-f_{1} f_{4} f_{22}+f_{2}{ }^{2} f_{3}-f_{2}{ }^{2} f_{33}+f_{2} f_{4} f_{1}-f_{2} f_{4} f_{11} .
\end{gathered}
$$

$m_{\tau_{1}(3)}=m_{\tau_{2}(4)}$ is equivalent to $g_{12}=0$. By direct computation $g_{12}=(s-t) g_{121}$, where $g_{121}$ is a polynomial with all positive terms. So $t$ must be equal to $s$ if $g_{12}=0$. But when $t=s, m\left(\tau_{1}\right)=m\left(\tau_{2}\right)$ because $\left(s\left(\tau_{1}\right), t\left(\tau_{1}\right)\right)=\left(s\left(\tau_{2}\right), t\left(\tau_{2}\right)\right)=(s, s)$. It contradicts $m\left(\tau_{1}\right) \neq m\left(\tau_{2}\right)$.

These two contradictions prove that $q\left(\tau_{1}\right) \nsim q\left(\tau_{2}\right)$.

(b) Because $m_{\tau_{1}(1)}=m_{\tau_{3}(1)}=m_{1}$ and $m_{\tau_{1}(2)}=m_{\tau_{3}(5-2)}=m_{2}, q\left(\tau_{1}\right) \nsim q\left(\tau_{3}\right)$.

(c) Because $m_{\tau_{1}(1)}=m_{\tau_{4}(1)}=m_{1}$ and $m_{\tau_{1}(3)}=m_{\tau_{4}(5-3)}=m_{3}, q\left(\tau_{1}\right) \nsim q\left(\tau_{4}\right)$.

(d) Because $m_{\tau_{1}(1)}=m_{\tau_{5}(1)}=m_{1}$ and $m_{\tau_{1}(2)}=m_{\tau_{5}(5-2)}=m_{2}, q\left(\tau_{1}\right) \nsim q\left(\tau_{5}\right)$.

(e) $q\left(\tau_{1}\right) \not q\left(\tau_{6}\right)$.

$\left(e_{1}\right)$ If $q\left(\tau_{1}\right) \sim q\left(\tau_{6}\right)$ and $\left(s\left(\tau_{1}\right), t\left(\tau_{1}\right)\right)=\left(s\left(\tau_{6}\right), t\left(\tau_{6}\right)\right)$, then $m\left(\tau_{1}\right)=m\left(\tau_{6}\right)$ because $m_{\tau_{1}(1)}=m_{\tau_{6}(1)}$ by Lemma 3.2. This contradicts $m\left(\tau_{1}\right) \neq m\left(\tau_{6}\right)$.

$\left(e_{2}\right)$ If $q\left(\tau_{1}\right) \sim q\left(\tau_{6}\right)$ and $\left(s\left(\tau_{1}\right), t\left(\tau_{1}\right)\right)=\left(t\left(\tau_{6}\right), s\left(\tau_{6}\right)\right)$, then $\lambda\left(\tau_{1}\right)=\lambda\left(\tau_{6}\right)$, $f_{i}\left(s\left(\tau_{1}\right), t\left(\tau_{1}\right)\right)=f_{i i}\left(s\left(\tau_{6}\right), t\left(\tau_{6}\right)\right)$. Using the fact that $m_{\tau_{1}(1)}=m_{\tau_{6}(1)}=m_{1}$ and 
$m_{\tau_{1}(2)}=m_{\tau_{6}(4)}=m_{2}$, we solve for $u\left(\tau_{1}\right)$ and $u\left(\tau_{6}\right)$,

$$
\begin{aligned}
& u\left(\tau_{1}\right)=\frac{\lambda\left(f_{2} f_{1}-f_{22} f_{1}-f_{2} f_{11}+f_{22} f_{3}\right)}{f_{2}{ }^{2}-f_{22} f_{4}}, \\
& u\left(\tau_{6}\right)=-\frac{\lambda\left(-f_{3} f_{2}-f_{4} f_{1}+f_{4} f_{11}+f_{2} f_{1}\right)}{f_{2}{ }^{2}-f_{22} f_{4}} .
\end{aligned}
$$

Substituting $u\left(\tau_{1}\right)$ into equations (22), we have

$$
\begin{aligned}
& m_{\tau_{1}(1)}=\frac{\lambda\left(f_{1} f_{22} f_{4}-f_{2} f_{22} f_{1}-f_{2}{ }^{2} f_{11}+f_{2} f_{22} f_{3}\right)}{\left(-f_{2}{ }^{2}+f_{22} f_{4}\right) f_{5}}, \\
& m_{\tau_{1}(2)}=-\frac{\lambda\left(f_{3} f_{2}{ }^{2}+f_{4} f_{2} f_{1}-f_{1} f_{22} f_{4}-f_{4} f_{2} f_{11}\right)}{\left(-f_{2}{ }^{2}+f_{22} f_{4}\right) f_{5}}, \\
& m_{\tau_{1}(3)}=\frac{\lambda\left(-f_{33} f_{2}{ }^{2}+f_{33} f_{22} f_{4}+f_{44} f_{2} f_{1}-f_{44} f_{22} f_{1}-f_{44} f_{2} f_{11}+f_{44} f_{22} f_{3}\right)}{\left(-f_{2}{ }^{2}+f_{22} f_{4}\right) f_{5}}, \\
& m_{\tau_{1}(4)}=\frac{\lambda\left(-f_{2}{ }^{2} f_{11}+f_{11} f_{22} f_{4}-f_{2} f_{22} f_{1}+f_{22}{ }^{2} f_{1}+f_{22} f_{2} f_{11}-f_{22}{ }^{2} f_{3}\right)}{\left(-f_{2}{ }^{2}+f_{22} f_{4}\right) f_{5}},
\end{aligned}
$$

which are the same as (27). $m_{\tau_{1}(3)}-m_{\tau_{6}(3)}=-\frac{\lambda g_{51}}{\left(-f_{2}^{2}+f_{22} f_{4}\right) f_{5}}$ and $m_{\tau_{1}(4)}-m_{\tau_{6}(2)}=$ $\frac{\lambda g_{51}}{\left(-f_{2}^{2}+f_{22} f_{4}\right) f_{5}}$, where $g_{51}$ is given by equation (28). $\cdot m_{\tau_{1}(3)}=m_{\tau_{6}(3)}$ and $m_{\tau_{1}(4)}=$ $m_{\tau_{6}(2)}$ are equivalent to $g_{51}=0$. However, $m_{\tau_{1}(1)}$ is always negative along the implicit curve $\Gamma_{51}$ on which $g_{51}=0$ (see Figure 3 and Proposition 3.3). This proves that $q\left(\tau_{1}\right) \nsim q\left(\tau_{6}\right)$.

(f) $q\left(\tau_{1}\right) \nsim q\left(\tau_{7}\right)$.

$\left(f_{1}\right)$ If $q\left(\tau_{1}\right) \sim q\left(\tau_{7}\right)$ and $\left(s\left(\tau_{1}\right), t\left(\tau_{1}\right)\right)=\left(s\left(\tau_{7}\right), t\left(\tau_{7}\right)\right)$, then by Lemma 3.2 $m\left(\tau_{1}\right)=m\left(\tau_{7}\right)$ because $m_{\tau_{1}(4)}=m_{\tau_{7}(4)}=m_{4}$. This contradicts $m\left(\tau_{1}\right) \neq m\left(\tau_{7}\right)$.

$\left(f_{2}\right)$ If $q\left(\tau_{1}\right) \sim q\left(\tau_{7}\right)$ and $\left(s\left(\tau_{1}\right), t\left(\tau_{1}\right)\right)=\left(t\left(\tau_{7}\right), s\left(\tau_{7}\right)\right)$, then $\lambda\left(\tau_{1}\right)=\lambda\left(\tau_{7}\right)$, $f_{i}\left(s\left(\tau_{1}\right), t\left(\tau_{1}\right)\right)=f_{i i}\left(s\left(\tau_{7}\right), t\left(\tau_{7}\right)\right)$. Using the fact that $m_{\tau_{1}(1)}=m_{\tau_{7}(2)}=m_{1}$ and $m_{\tau_{1}(2)}=m_{\tau_{7}(1)}=m_{2}$, we solve for $u\left(\tau_{1}\right)$ and $u\left(\tau_{7}\right)$,

$$
\begin{aligned}
& u\left(\tau_{1}\right)=\frac{\lambda\left(f_{44} f_{3}-f_{22} f_{33}-f_{44} f_{11}+f_{22} f_{1}\right)}{-f_{44} f_{4}+f_{22} f_{2}}, \\
& u\left(\tau_{7}\right)=-\frac{\lambda\left(f_{1} f_{4}+f_{2} f_{3}-f_{2} f_{11}-f_{33} f_{4}\right)}{-f_{44} f_{4}+f_{22} f_{2}} .
\end{aligned}
$$

$m_{\tau_{1}(3)}-m_{\tau_{7}(3)}=-\frac{\lambda g_{17}}{\left(-f_{44} f_{4}+f_{22} f_{2}\right) f_{5}}$, where

$$
\begin{aligned}
g_{17}= & -f_{33} f_{2} f_{22}+f_{33} f_{4} f_{44}+f_{44} f_{1} f_{22}-f_{44} f_{33} f_{22}+f_{44}{ }^{2} f_{3}-f_{4} 4^{2} f_{11} \\
& +f_{3} f_{2} f_{22}-f_{3} f_{4} f_{44}+f_{4} f_{2} f_{3}-f_{4} f_{2} f_{11}+f_{4}{ }^{2} f_{1}-f_{4}{ }^{2} f_{33} .
\end{aligned}
$$

So $m_{\tau_{1}(3)}=m_{\tau_{7}(3)}=m_{3}$ is equivalent to $g_{17}=0$. By direct computation $g_{17}=(s-t) g_{171}$, where $g_{171}$ is a polynomial with all positive terms. So $t$ must be equal to $s$ if $g_{17}=0$. But when $t=s, m\left(\tau_{1}\right)=m\left(\tau_{7}\right)$ because $\left(s\left(\tau_{1}\right), t\left(\tau_{1}\right)\right)=$ $\left(s\left(\tau_{7}\right), t\left(\tau_{7}\right)\right)=(s, s)$. It contradicts $m\left(\tau_{1}\right) \neq m\left(\tau_{7}\right)$.

These two contradictions show that $q\left(\tau_{1}\right) \nsim q\left(\tau_{7}\right)$.

(g) $q\left(\tau_{1}\right) \nsim q\left(\tau_{8}\right)$.

$\left(g_{1}\right)$ If $q\left(\tau_{1}\right) \sim q\left(\tau_{8}\right)$ and $\left(s\left(\tau_{1}\right), t\left(\tau_{1}\right)\right)=\left(s\left(\tau_{8}\right), t\left(\tau_{8}\right)\right)$, we have

$$
\lambda f_{1}-f_{2} u\left(\tau_{1}\right)=\lambda f_{3}+f_{4} u\left(\tau_{8}\right), \quad \lambda f_{3}+f_{4} u\left(\tau_{1}\right)=\lambda f_{1}-f_{2} u\left(\tau_{8}\right)
$$


because $m_{\tau_{1}(1)}=m_{\tau_{8}(1)}=m_{1}$ and $m_{\tau_{1}(2)}=m_{\tau_{8}(2)}=m_{2}$. Adding the two equations and rearranging the terms, we have $\left(-f_{2}+f_{4}\right) u\left(\tau_{1}\right)=\left(-f_{2}+f_{4}\right) u\left(\tau_{8}\right)$ which implies that $u\left(\tau_{1}\right)=u\left(\tau_{8}\right)$ because $f_{2}>f_{4}>0$ for any $s>0, t>0$. Then $m\left(\tau_{1}\right)=m\left(\tau_{8}\right)$, which contradicts $m\left(\tau_{1}\right) \neq m\left(\tau_{8}\right)$.

$\left(g_{2}\right)$ If $q\left(\tau_{1}\right) \sim q\left(\tau_{8}\right)$ and $\left(s\left(\tau_{1}\right), t\left(\tau_{1}\right)\right)=\left(t\left(\tau_{8}\right), s\left(\tau_{8}\right)\right)$, then $\lambda\left(\tau_{1}\right)=\lambda\left(\tau_{8}\right)$, $f_{i}\left(s\left(\tau_{1}\right), t\left(\tau_{1}\right)\right)=f_{i i}\left(s\left(\tau_{8}\right), t\left(\tau_{8}\right)\right)$. Using the fact that $m_{\tau_{1}(1)}=m_{\tau_{8}(2)}=m_{1}$ and $m_{\tau_{1}(2)}=m_{\tau_{8}(1)}=m_{2}$, we solve for $u\left(\tau_{1}\right)$ and $u\left(\tau_{8}\right)$,

$$
u\left(\tau_{1}\right)=\frac{\lambda g_{181}}{-f_{44} f_{4}+f_{22} f_{2}}, u\left(\tau_{8}\right)=-\frac{\lambda g_{182}}{-f_{44} f_{4}+f_{22} f_{2}},
$$

where

$$
\begin{aligned}
& g_{181}=\left(f_{44} f_{3}-f_{22} f_{33}-f_{44} f_{11}+f_{22} f_{1}\right), \\
& g_{182}=\left(f_{1} f_{4}+f_{2} f_{3}-f_{2} f_{11}-f_{33} f_{4}\right) .
\end{aligned}
$$

$m_{\tau_{1}(3)}-m_{\tau_{8}(4)}=\frac{\lambda\left(f_{2}+f_{44}\right)\left(g_{181}-g_{182}\right)}{\left(-f_{44} f_{4}+f_{22} f_{2}\right) f_{5}} \cdot m_{\tau_{1}(3)}=m_{\tau_{8}(4)}$ is equivalent to $g_{181}=g_{182}$, which implies $u\left(\tau_{8}\right)=-u\left(\tau_{1}\right)$. Therefore $m\left(\tau_{1}\right)=m\left(\operatorname{con}\left(\tau_{8}\right)\right)$, which contradicts $m\left(\tau_{1}\right) \neq m\left(\operatorname{con}\left(\tau_{8}\right)\right)$.

(h) Because $m_{\tau_{1}(4)}=m_{\tau_{9}(4)}=m_{4}$ and $m_{\tau_{1}(3)}=m_{\tau_{9}(5-3)}=m_{3}, q\left(\tau_{1}\right) \nsim q\left(\tau_{9}\right)$.

(i) Because $m_{\tau_{1}(4)}=m_{\tau_{11}(4)}=m_{4}$ and $m_{\tau_{1}(2)}=m_{\tau_{11}(5-2)}=m_{2}, q\left(\tau_{1}\right) \nsim q\left(\tau_{11}\right)$.

(j) $q\left(\tau_{1}\right) \nsim q\left(\tau_{12}\right)$.

$\left(j_{1}\right)$ If $q\left(\tau_{1}\right) \sim q\left(\tau_{12}\right)$ and $\left(s\left(\tau_{1}\right), t\left(\tau_{1}\right)\right)=\left(s\left(\tau_{12}\right), t\left(\tau_{12}\right)\right), m\left(\tau_{1}\right)=m\left(\tau_{12}\right)$ because $m_{\tau_{1}(4)}=m_{\tau_{12}(4)}=m_{4}$. This contradicts $m\left(\tau_{1}\right) \neq m\left(\tau_{12}\right)$.

$\left(j_{2}\right)$ If $q\left(\tau_{1}\right) \sim q\left(\tau_{12}\right)$ and $\left(s\left(\tau_{1}\right), t\left(\tau_{1}\right)\right)=\left(t\left(\tau_{12}\right), s\left(\tau_{12}\right)\right)$, then $\lambda\left(\tau_{1}\right)=\lambda\left(\tau_{12}\right)$, $f_{i}\left(s\left(\tau_{1}\right), t\left(\tau_{1}\right)\right)=f_{i i}\left(s\left(\tau_{12}\right), t\left(\tau_{12}\right)\right)$. Using the fact that $m_{\tau_{1}(1)}=m_{\tau_{12}(3)}=m_{1}$ and $m_{\tau_{1}(2)}=m_{\tau_{12}(2)}=m_{2}$, we solve for $u\left(\tau_{1}\right)$ and $u\left(\tau_{12}\right)$,

$$
\begin{aligned}
& u\left(\tau_{1}\right)=\frac{\lambda\left(f_{1} f_{44}-f_{3} f_{44}+f_{4} f_{3}-f_{4} f_{33}\right)}{f_{2} f_{44}-f_{4}{ }^{2}}, \\
& u\left(\tau_{12}\right)=-\frac{\lambda\left(f_{2} f_{3}-f_{2} f_{33}+f_{4} f_{1}-f_{4} f_{3}\right)}{f_{2} f_{44}-f_{4}{ }^{2}} .
\end{aligned}
$$

Substituting $u\left(\tau_{1}\right)$ into equations (22) we have

$$
\begin{aligned}
& m_{\tau_{1}(1)}=-\frac{\lambda\left(f_{1} f_{4}{ }^{2}-f_{2} f_{3} f_{44}+f_{4} f_{2} f_{3}-f_{2} f_{4} f_{33}\right)}{\left(f_{2} f_{44}-f_{4}{ }^{2}\right) f_{5}}, \\
& m_{\tau_{1}(2)}=\frac{\lambda\left(f_{2} f_{3} f_{44}+f_{1} f_{44} f_{4}-f_{44} f_{4} f_{3}-f_{33} f_{4}{ }^{2}\right)}{\left(f_{2} f_{44}-f_{4}{ }^{2}\right) f_{5}}, \\
& m_{\tau_{1}(3)}=-\frac{\lambda\left(-f_{33} f_{2} f_{44}+f_{33} f_{4}{ }^{2}+f_{1} f_{44}{ }^{2}-f_{3} f_{44}{ }^{2}+f_{44} f_{4} f_{3}-f_{44} f_{4} f_{33}\right)}{\left(f_{2} f_{44}-f_{4}{ }^{2}\right) f_{5}}, \\
& m_{\tau_{1}(4)}=\frac{\lambda\left(f_{11} f_{2} f_{44}-f_{11} f_{4}{ }^{2}+f_{22} f_{1} f_{44}-f_{22} f_{3} f_{44}+f_{22} f_{4} f_{3}-f_{22} f_{4} f_{33}\right)}{\left(f_{2} f_{44}-f_{4}{ }^{2}\right) f_{5}},
\end{aligned}
$$

and $m_{\tau_{1}(3)}-m_{\tau_{12}(1)}=\frac{\lambda g_{112}}{\left(f_{2} f_{44}-f_{4}^{2}\right) f_{5}}$, where

$$
\begin{gathered}
g_{112}=f_{33} f_{2} f_{44}-f_{33} f_{4}^{2}-f_{1} f_{44}^{2}+f_{3} f_{44}^{2}-f_{44} f_{4} f_{3}+f_{44} f_{4} f_{33}-f_{11} f_{2} f_{44} \\
+f_{11} f_{4}^{2}+f_{22} f_{2} f_{3}-f_{22} f_{2} f_{33}+f_{22} f_{4} f_{1}-f_{22} f_{4} f_{3} .
\end{gathered}
$$


Further, note that for $g_{51}$ given by equation (28),

$g_{51}+g_{112}=\left(f_{2}+f_{44}-f_{4}-f_{22}\right)\left(-f_{3} f_{2}+f_{2} f_{33}-f_{44} f_{1}-f_{4} f_{1}+f_{44} f_{3}+f_{33} f_{4}\right)$. So $g_{112} \equiv g_{51}$ because $\left(f_{2}+f_{44}-f_{4}-f_{22}\right) \equiv 0$ for all $s, t$. So $m_{\tau_{1}(3)}=m_{\tau_{12}(1)}$ is equivalent to $g_{51}=0$. In addition, $m_{\tau_{1}(4)}$ in (38) is the same as $m_{\tau_{2}(1)}$ in (27) on the implicit curve $g_{51}=0$ because

$$
m_{\tau_{1}(4)} \text { in (38) }-m_{\tau_{2}(1)} \text { in (27) }=\frac{\lambda f_{22} f_{4} g_{51}}{\left(f_{2} f_{44}-f_{4}^{2}\right)\left(f_{2}^{2}-f_{22} f_{4}\right) f_{5}} .
$$

Then $m_{\tau_{1}(4)}$ in (38) is always negative along the implicit curve $\Gamma_{51}$ (see Figure 3) by Proposition 3.3 So we have proved that $q\left(\tau_{1}\right) \nsim q\left(\tau_{12}\right)$.

We have proved that $q\left(\tau_{1}\right) \not q\left(\tau_{i}\right)$ for $i \in\{1,2, \cdots, 12\} \backslash\{1,10\}$. We can apply the same arguments to the other cases. We omit the proof here.

Proof of Lemma 3.6. We first prove that $q\left(\tau_{1}\right) \sim q\left(\tau_{10}\right)$ if and only if $m=\left(m_{1}, m_{2}\right.$, $\left.m_{3}, m_{4}\right) \in \Psi_{1}$.

If $q\left(\tau_{1}\right) \sim q\left(\tau_{10}\right)$ and $\left(s\left(\tau_{1}\right), t\left(\tau_{1}\right)\right)=\left(s\left(\tau_{10}\right), t\left(\tau_{10}\right)\right)$, then $\lambda\left(\tau_{1}\right)=\lambda\left(\tau_{10}\right)$. Using the fact that $m_{\tau_{1}(1)}=m_{\tau_{10}(3)}$ and $m_{\tau_{1}(2)}=m_{\tau_{10}(1)}$, we solve for $u\left(\tau_{1}\right)$ and $u\left(\tau_{10}\right)$,

$$
\begin{aligned}
& u\left(\tau_{1}\right)=\frac{\lambda\left(f_{2} f_{1}+f_{44} f_{1}-f_{2} f_{33}-f_{44} f_{3}\right)}{f_{2}{ }^{2}+f_{44} f_{4}}, \\
& u\left(\tau_{10}\right)=\frac{\lambda\left(f_{2} f_{1}+f_{4} f_{33}-f_{2} f_{3}-f_{4} f_{1}\right)}{f_{2}{ }^{2}+f_{44} f_{4}} .
\end{aligned}
$$

Substituting $u\left(\tau_{1}\right)$ into equations (22), we have

$$
\begin{aligned}
& m_{\tau_{1}(1)}=\frac{\lambda\left(f_{1} f_{44} f_{4}-f_{2} f_{44} f_{1}+f_{2}{ }^{2} f_{33}+f_{2} f_{44} f_{3}\right)}{\left(f_{2}{ }^{2}+f_{44} f_{4}\right) f_{5}}, \\
& m_{\tau_{1}(2)}=\frac{\lambda\left(f_{3} f_{2}{ }^{2}+f_{4} f_{2} f_{1}+f_{1} f_{44} f_{4}-f_{4} f_{2} f_{33}\right)}{\left(f_{2}{ }^{2}+f_{44} f_{4}\right) f_{5}}, \\
& m_{\tau_{1}(3)}=\frac{\lambda\left(f_{2}{ }^{2} f_{33}+f_{33} f_{44} f_{4}-f_{2} f_{44} f_{1}-f_{44}{ }^{2} f_{1}+f_{44} f_{2} f_{33}+f_{44}{ }^{2} f_{3}\right)}{\left(f_{2}{ }^{2}+f_{44} f_{4}\right) f_{5}}, \\
& m_{\tau_{1}(4)}=-\frac{\lambda\left(-f_{11} f_{2}{ }^{2}-f_{11} f_{44} f_{4}-f_{22} f_{2} f_{1}-f_{22} f_{44} f_{1}+f_{22} f_{2} f_{33}+f_{22} f_{44} f_{3}\right)}{\left(f_{2}{ }^{2}+f_{44} f_{4}\right) f_{5}}
\end{aligned}
$$

which means that $m_{i}=m_{\tau_{1}(i)}=\varphi_{i}$ for $i=1,2,3,4$, by comparing with equation (16). $m_{\tau_{1}(3)}-m_{\tau_{10}(4)}=-\frac{\lambda g_{31}}{\left(f_{2}{ }^{2}+f_{44} f_{4}\right) f_{5}}$ and $m_{\tau_{1}(4)}-m_{\tau_{10}(2)}=\frac{\lambda g_{32}}{\left(f_{2}{ }^{2}+f_{44} f_{4}\right) f_{5}}$, where $g_{31}$ is given by (11) and

$$
\begin{aligned}
g_{32}=- & -f_{11} f_{2}^{2}-f_{11} f_{44} f_{4}-f_{22} f_{2} f_{1}-f_{22} f_{44} f_{1}+f_{22} f_{2} f_{33}+f_{22} f_{44} f_{3} \\
& \left.+f_{3} f_{2}{ }^{2}+f_{3} f_{44} f_{4}-f_{4} f_{3} f_{2}-f_{4}^{2} f_{1}+f_{4}^{2} f_{33}+f_{4} f_{2} f_{1}\right) .
\end{aligned}
$$

Then $m_{\tau_{1}(3)}=m_{\tau_{10}(4)}=m_{3}$ and $m_{\tau_{1}(4)}=m_{\tau_{10}(2)}=m_{4}$ are equivalent to $g_{31}=0$ and $g_{32}=0$. Note that

$g_{31}-g_{32}=-\left(f_{2}-f_{4}+f_{44}-f_{22}\right)\left(f_{3} f_{2}-f_{2} f_{33}+f_{4} f_{1}+f_{44} f_{1}-f_{44} f_{3}-f_{4} f_{33}\right)$ and that $\left(f_{2}-f_{4}+f_{44}-f_{22}\right)=0$ for all $s, t$ by Proposition 2.1. Then $g_{31}=g_{32}$ for all $s, t$. By the properties of $\Gamma_{31}$ and $\bar{\Gamma}_{31}$ in Proposition 2.5, if $q\left(\tau_{1}\right) \sim q\left(\tau_{10}\right)$ and $\left(s\left(\tau_{1}\right), t\left(\tau_{1}\right)\right)=\left(s\left(\tau_{10}\right), t\left(\tau_{10}\right)\right)$, then $m=m\left(\tau_{1}\right)=\left(\varphi_{1}, \varphi_{2}, \varphi_{3}, \varphi_{4}\right)$ given by (40) is mutually distinct positive and $m \in \psi_{11}$.

Conversely, if $m \in \psi_{11}$, then $m\left(\tau_{1}\right)=\left(\varphi_{1}, \varphi_{2}, \varphi_{3}, \varphi_{4}\right)(\lambda, s)$ for some $0<\lambda<\infty$ and $s_{0}<s<s_{1}, s \neq \bar{s}$. By Proposition [2.5, there exists a unique $t=t(s)$ such 
that $g_{31}(s, t(s))=0$. For these values $\lambda, s, t(s)$, we can find $u\left(\tau_{1}\right)$ and $u\left(\tau_{10}\right)$ by equation (39). Then the central configuration for $m\left(\tau_{1}\right)$ is equivalent to the central configuration for $m\left(\tau_{10}\right)$ because $q\left(\tau_{1}\right)=q\left(\tau_{10}\right)=(-s-1,-1,1, t(s)+1)$.

If $q\left(\tau_{1}\right) \sim q\left(\tau_{10}\right)$ and $\left(s\left(\tau_{1}\right), t\left(\tau_{1}\right)\right)=\left(t\left(\tau_{10}\right), s\left(\tau_{10}\right)\right)$, then $\lambda\left(\tau_{1}\right)=\lambda\left(\tau_{10}\right)$ and $f_{i}\left(s\left(\tau_{1}\right), t\left(\tau_{1}\right)\right)=f_{i i}\left(s\left(\tau_{10}\right), t\left(\tau_{10}\right)\right)$. Using the fact that $m_{\tau_{1}(1)}=m_{\tau_{10}(2)}=m_{1}$ and $m_{\tau_{1}(2)}=m_{\tau_{10}(1)}=m_{2}$, we solve for $u\left(\tau_{1}\right)$ and $u\left(\tau_{10}\right)$,

$$
\begin{aligned}
& u\left(\tau_{1}\right)=\frac{\lambda\left(f_{1} f_{22}-f_{3} f_{22}-f_{4} f_{3}+f_{4} f_{11}\right)}{f_{2} f_{22}+f_{4}{ }^{2}}, \\
& u\left(\tau_{10}\right)=-\frac{\lambda\left(f_{2} f_{3}-f_{2} f_{11}+f_{4} f_{1}-f_{4} f_{3}\right)}{f_{2} f_{22}+f_{4}{ }^{2}} .
\end{aligned}
$$

Substituting $u\left(\tau_{1}\right)$ into equations (22), we have

$$
\begin{aligned}
& m_{\tau_{1}(1)}=\frac{\lambda\left(f_{1} f_{4}{ }^{2}+f_{2} f_{3} f_{22}+f_{2} f_{4} f_{3}-f_{2} f_{4} f_{11}\right)}{\left(f_{2} f_{22}+f_{4}\right) f_{5}}, \\
& m_{\tau_{1}(2)}=\frac{\lambda\left(f_{2} f_{3} f_{22}+f_{4} f_{1} f_{22}-f_{4} f_{3} f_{22}+f_{4}{ }^{2} f_{11}\right)}{\left(f_{2} f_{22}+f_{4}{ }^{2}\right) f_{5}}, \\
& m_{\tau_{1}(3)}=\frac{\lambda\left(f_{33} f_{2} f_{22}+f_{33} f_{4}{ }^{2}-f_{44} f_{1} f_{22}+f_{44} f_{3} f_{22}+f_{44} f_{4} f_{3}-f_{44} f_{4} f_{11}\right)}{\left(f_{2} f_{22}+f_{4}{ }^{2}\right) f_{5}}, \\
& m_{\tau_{1}(4)}=\frac{\lambda\left(f_{11} f_{2} f_{22}+f_{4}{ }^{2} f_{11}+f_{1} f_{22}{ }^{2}-f_{3} f_{22}{ }^{2}-f_{4} f_{3} f_{22}+f_{22} f_{4} f_{11}\right)}{\left(f_{2} f_{22}+f_{4}{ }^{2}\right) f_{5}} .
\end{aligned}
$$

Note that $m_{\tau_{1}(1)}-\varphi_{2}=\frac{\lambda f_{4} f_{2} g_{31}}{\left(f_{4}{ }^{2}+f_{22} f_{2}\right) f_{5}\left(f_{4} f_{44}+f_{2}{ }^{2}\right)}$, which means that $m_{\tau_{1}(1)}=\varphi_{2}$ along the curve $g_{31}=0$. Similarly, we have $m_{\tau_{1}(2)}=\varphi_{4}, m_{\tau_{1}(3)}=\varphi_{1}$, and $m_{\tau_{1}(4)}=$ $\varphi_{3}$ along the curve $g_{31}=0$. So $m_{i}=m_{\tau_{1}(i)}=\varphi_{\tau_{10}(i)}$ for $i=1,2,3,4$.

By direct computation, $m_{\tau_{1}(3)}-m_{\tau_{10}(1)}=\frac{\lambda g_{91}}{\left(f_{2} f_{22}+f_{4}{ }^{2}\right) f_{5}}$ and $m_{\tau_{1}(4)}-m_{\tau_{10}(3)}=$ $-\frac{\lambda g_{92}}{\left(f_{2} f_{22}+f_{4}{ }^{2}\right) f_{5}}$, where

$$
\begin{gathered}
g_{91}=f_{33} f_{2} f_{22}+f_{33} f_{4}{ }^{2}-f_{44} f_{1} f_{22}+f_{44} f_{3} f_{22}+f_{44} f_{4} f_{3}-f_{44} f_{4} f_{11} \\
-f_{1} f_{2} f_{22}-f_{1} f_{4}{ }^{2}+f_{2}{ }^{2} f_{3}-f_{2}{ }^{2} f_{11}+f_{2} f_{4} f_{1}-f_{2} f_{4} f_{3}, \\
g_{92}=-f_{11} f_{2} f_{22}-f_{4}{ }^{2} f_{11}-f_{1}{f_{22}}^{2}+f_{3}{f_{22}}^{2}+f_{4} f_{3} f_{22}-f_{22} f_{4} f_{11}+f_{33} f_{2} f_{22} \\
+f_{33} f_{4}{ }^{2}-f_{44} f_{2} f_{3}+f_{44} f_{2} f_{11}-f_{44} f_{4} f_{1}+f_{44} f_{4} f_{3} .
\end{gathered}
$$

$m_{\tau_{1}(3)}=m_{\tau_{10}(1)}$ and $m_{\tau_{1}(4)}=m_{\tau_{10}(3)}$ are equivalent to $g_{91}=0$ and $g_{92}=0$. Note that

$$
g_{91}-g_{92}=\left(f_{2}-f_{4}+f_{44}-f_{22}\right)\left(f_{2} f_{3}-f_{2} f_{11}-f_{1} f_{22}+f_{4} f_{1}+f_{3} f_{22}-f_{4} f_{11}\right)
$$

and that $\left(f_{2}-f_{4}+f_{44}-f_{22}\right)=0$ for all $s>, t$. Then $g_{91}=g_{92}$ for all $s, t$. We further note that

$$
\begin{aligned}
& g_{31}-g_{91}=\left(f_{2}-f_{4}+f_{44}-f_{22}\right) \\
& \times\left(-f_{2} f_{3}+f_{2} f_{33}-f_{44} f_{1}-f_{4} f_{1}+f_{44} f_{3}+f_{33} f_{4}\right) .
\end{aligned}
$$

Then $g_{91}=g_{31}$ for all $s>0, t>0$ because $f_{2}-f_{4}+f_{44}-f_{22} \equiv 0$. By the properties of $\Gamma_{31}$ and $\bar{\Gamma}_{31}$ in Proposition 2.5 if $q\left(\tau_{1}\right) \sim q\left(\tau_{10}\right)$ and $\left(s\left(\tau_{1}\right), t\left(\tau_{1}\right)\right)=$ $\left(t\left(\tau_{10}\right), s\left(\tau_{10}\right)\right)$, then $m=m\left(\tau_{1}\right)=\left(\varphi_{2}, \varphi_{4}, \varphi_{1}, \varphi_{3}\right)$ given by (40) is mutually distinct positive and $m \in \psi_{12}$.

Conversely, if $m \in \psi_{12}$, then $m\left(\tau_{1}\right)=\left(\varphi_{2}, \varphi_{4}, \varphi_{1}, \varphi_{3}\right)(\lambda, s)$ for some $0<\lambda<\infty$ and $s_{0}<s<s_{1}, s \neq \bar{s}$. By Proposition 2.5] there exists a unique $t=t(s)$ such that $g_{31}(s, t(s))=0$. For these values $\lambda, s, t(s)$, we can find $u\left(\tau_{1}\right)$ and 
$u\left(\tau_{10}\right)$ by equation (41). Then the central configuration for $m\left(\tau_{1}\right)$ is equivalent to the central configuration for $m\left(\tau_{10}\right)$ because $q\left(\tau_{1}\right)=(-s-1,-1,1, t(s)+1)$ and $q\left(\tau_{10}\right)=(-t(s)-1,-1,1, s+1)$.

So we have proved that $q\left(\tau_{1}\right) \sim q\left(\tau_{10}\right)$ if and only if $m=\left(m_{1}, m_{2}, m_{3}, m_{4}\right) \in \Psi_{1}$. By the same arguments, we can prove the other cases and we omit the proofs.

Proof of Lemma 3.7. If $m_{1}, m_{2}, m_{3}, m_{4}$ are mutually distinct positive masses and if $q\left(\tau_{i}\right) \sim q\left(\tau_{j}\right)$ and $q\left(\tau_{k}\right) \sim q\left(\tau_{l}\right)$, then $(i, j)$ and $(k, l)$ can be chosen only from the set $\{(1,10),(2,9),(3,8),(4,12),(5,7),(6,11)\}$ by Lemma 3.6. If $q\left(\tau_{i}\right) \sim q\left(\tau_{j}\right)$ and $q\left(\tau_{k}\right) \sim q\left(\tau_{l}\right)$ hold simultaneously and $(i, j)$ and $(k, l)$ are chosen from the above set, then $m=\left(m_{1}, m_{2}, m_{3}, m_{4}\right) \in \Psi_{i}$ and $m=\left(m_{1}, m_{2}, m_{3}, m_{4}\right) \in \Psi_{k}$ simultaneously. However, by the definition of $\Psi_{i}, \varphi_{i}$ in (16), and Proposition 2.5, we know that $\Psi_{i} \bigcap \Psi_{k}$ for $1 \leq i \neq k \leq 6$ is an empty set. So no mass vector $\left(m_{1}, m_{2}, m_{3}, m_{4}\right)$ can make $q\left(\tau_{i}\right) \sim q\left(\tau_{j}\right)$ and $q\left(\tau_{k}\right) \sim q\left(\tau_{l}\right)$ hold simultaneously for $1 \leq i, j, k, l \leq 12$ and $i, j, k, l$ are mutually distinct.

\section{ACKNOWLEDGEMENTS}

The authors would like to thank the editors and the anonymous referees for their comments and suggestions regarding this work. The referees' suggestions helped improve the clarity and the overall presentation of the results.

\section{REFERENCES}

[1] A. Albouy, Symetrie des configurations centrales de quatre corps (French) [Symmetry of central configurations of four bodies] C. R. Acad. Sci. Paris Soc. I Math. 320 (1995), no. 2, 217-220. MR.1320359 (95k:70023)

[2] A. Albouy, The symmetric central configurations of four equal masses. Hamiltonian dynamics and celestial mechanics (Seattle, WA, 1995), 131-135, Contemp. Math., 198, Amer. Math. Soc., Providence, RI, 1996. MR1409157 (97g:70012)

[3] A. Albouy, Y. Fu and S. Sun, Symmetry of planar four-body convex central configurations, Proc. Royal Soc. A 464 (2008), 1355-1365. MR2386651 (2009d:70012)

[4] A. Albouy and R. Moeckel, The inverse problem for collinear central configuration, Celestial Mechanics and Dynamical Astronomy 77 (2000), 77-91. MR.1820352 (2002b:70017)

[5] M. Arribas, A. Elipe, T. Kalvouridis, and M. Palacios, Homographic solutions in the planar $n+1$ body problem with quasi-homogeneous potentials, Celestial Mech. Dyn. Astr. 99 (2007), 1-12. MR2347831 (2010a:70014)

[6] M. Hampton and R. Moeckel, Finiteness of relative equilibria of the four-body problem. Invent. Math. 163 (2006), no. 2, 289-312. MR2207019 (2008c:70019)

[7] M. Hampton and M. Santoprete, Seven-body central configurations: A family of central configurations in the spatial seven-body problem, Celestial Mech. Dyn. Astr. 99 (2007), 293305. MR2441568 (2009e:70031)

[8] J. Llibre and L.F. Mello, New central configurations for the planar 5-body problem, Celestial Mech. Dyn. Astr. 100 (2008), 141-149. MR2384337

[9] J. Lee and M. Santoprete, Central configurations of the five-body problem with equal masses, Celestial Mech. Dyn. Astr. 104 (2009), 369-381. MR2524814(2010i:70007)

[10] T. Lei and M. Santoprete, Rosette central configurations, degenerate central configurations and bifurcations, Celestial Mech. Dyn. Astr. 94 (2006), 271-287. MR2247661(2008d:70021)

[11] N. Jacobson, Basic Algebra I. W.H. Freeman and Co., 1974. MR0356989 (50:9457)

[12] J. Bernat, J. Llibre, and E. Perez-Chavela, On the planar central configurations of the 4-body problem with three equal masses. Dynamics of Continuous, Discrete and Impulsive Systems Series A: Mathematical Analysis 16 (2009), 1-13. MR2488967|(2010b:70017)

[13] E. Leandro, Finiteness and bifurcations of some symmetrical classes of central configurations. Arch. Ration. Mech. Anal. 167 (2003), no. 2, 147-177. MR.1971151(2004c:70022) 
[14] E. Leandro, On the central configurations of the planar restricted four-body problem. J. Differential Equations 226 (2006), no. 1, 323-351. MR2232439(2008a:70022)

[15] Y. Long and S. Sun, Collinear central configurations and singular surfaces in the mass space, Arch. Rational Mech. Anal. 173 (2004), 151-167. MR2081029 (2006d:70033)

[16] Y. Long and S. Sun, "Collinear central configurations in celestial mechanics", Topological Methods, Variational Methods and Their Applications: ICM 2002 Satellite Conference on Nonlinear Functional Analysis, Taiyuan, Shan Xi, P.R. China, August 14 - 18, 2002 By H. Brezis, K. C. Chang, pp. 159-165. MR2011693 (2004i:70012)

[17] Y. Long and S. Sun, Four-body central configurations with some equal masses, Arch. Ration. Mech. Anal. 162 (2002), no. 1, 25-44. MR.1892230 (2003a:70013)

[18] K. Meyer and G. R. Hall, "Introduction to Hamiltonian Dynamical System and the N-Body Problem", Applied Mathematical Sciences, Vol. 90, Springer, 1992. MR.1140006 (93b:70002)

[19] K. Meyer, G. R. Hall and D. Offin, "Introduction to Hamiltonian Dynamical System and the N-Body Problem", second edition, Applied Mathematical Sciences, Vol. 90, Springer, 2009. MR 2468466 (2010d:37108)

[20] F.R. Moulton, The straight line solutions of the problem of N bodies, Ann. Math., II. Ser. 12, 1910, 1-17. MR.1503509

[21] R. Moeckel, On central configurations, Math. Zeit. 205 (1990), 499-517. MR1082871 (92b:70012)

[22] T. Ouyang and Z. Xie, Collinear central confiugration in four-body problem, Cele. Mech. \& Dyna. Astr. 93 (2005) 147-166. MR.2186805 (2006h:70016)

[23] E. Perez-Chavela and M. Santoprete, Convex four-body central configurations with some equal masses, Arch. Ration. Mech. Anal. 185 (2007), no. 3, 481-494. MR 2322818 (2008m:70030)

[24] D. Saari, On the role and the properties of $n$-body central configurations, Clestial Mech. 21 (1980), 9-20. MR 564603 (81a:70016)

[25] J. Shi and Z. Xie, Classification of four-body central configurations with three equal masses, Journal of Mathematical Analysis and Applications 363 (2010), 512-524. MR2564872

[26] S. Smale, Topology and mechanics. II. The planar n-body problem, Invent. Math. 11 (1970), 45-64. MR0321138 (47:9671)

[27] S. Smale, Mathematical problems for the next century, the Mathematical Intelligencer, Volume 20, Number 2, 1998. MR1631413 (99h:01033)

[28] D. Schmidt, Central configurations in $\mathbf{R}^{2}$ and $\mathbf{R}^{3}$. Contemp. Math. 81 (1988) 59-76. MR986257 (90d:70028)

[29] W.L. Williams, Permanent Configurations in the Problem of Five Bodies, Trans. Amer. Math. Soc. 44 (1938), 563-579. MR1501982

[30] A. Wintner, The analytical Foundations of Celestial Mechanics. Princeton Math. Series 5, 215. Princeton Univ. Press, Princeton, NJ, 1941. MR0005824 (3:215b)

[31] M. Woodlin and Z. Xie, Collinear central configurations in the $n$-body problem with general homogeneous potential, Jour. Math. Physics 50 (2009), 102901. MR2573123

[32] Z. Xia, Convex central configurations for the $n$-body problem. J. Differential Equations 200 (2004), no. 2, 185-190. MR2052612 (2005f:70018)

[33] Z. Xie, Inverse problem of central configurations and singular curve in the collinear 4-body problem, Cele. Mech. \& Dyna. Astr. 107 (2010), 353-376.

[34] Z. Xie, Super central configurations of the $n$-body problem Jour. Math. Physics 51 (2010), 042902, 7 pp.

[35] Z. Xie, Central Configurations of Collinear Three-body Problem and Singular Surfaces in the Mass Space, Phys. Lett. A. 375 (2011) no. 39, 3392-3398. MR2826168

[36] S. Zhang, Two layer nested regular polygon central configurations in $\mathbf{R}^{3}$, Phys. Lett. A, 290 (2001) no. 1, 49-54. MR1876951(2002j:70013)

Department of Mathematics, Brigham Young University, Provo, Utah 84602

Department of Mathematics and Computer Science, Virginia State University, PeTERSBURG, VIRGINIA 23806

E-mail address: zxie@vsu.edu 